\title{
SYNTHESIS, MOLECULAR DOCKING AND ANTI-PROLIFERATIVE ACTIVITY OF NEW SERIES OF 1-METHYLSULPHONYL-3-INDOLYL HETEROCYCLES
}

\author{
HEBA M. ABO-SALEM ${ }^{1}$, KHADIGA M. AHMED ${ }^{1}$, SALWA EL-HALLOUTY², ESLAM R. EL-SAWY1*, \\ ADEL H. MANDOUR ${ }^{1}$
}

\author{
${ }^{1}$ Chemistry Department of Natural Compounds, National Research Centre, 12311 Dokki, Giza, Egypt, ${ }^{2}$ Pharmacognosy Department, \\ National Research Centre, 12311 Dokki, Giza, Egypt \\ Email: erelsawynrc@gmail.com
}

Received: 23 Aug 2016 Revised and Accepted: 05 0ct 2016

\begin{abstract}
Objective: The present work aimed to synthesize a new series of 1-methylsulphonyl-3-indolyl heterocycles and study their cytotoxic activity. In addition, we attempted to explore the mode of the interaction of anti-proliferative compounds with the active site of carbonic anhydrase IX (CA IX) theoretically via molecular docking study.

Methods: Novel series of pyrazole, pyrimidine and triazole derivatives bearing 1-methylsulphonyl-1H-indole were prepared via a series of hetero cyclization reactions utilizing 3-(1-methylsulphonyl-1H-indol-3-yl)-1-(substituted phenyl)-1H-pyrazole-4-carboxaldehydes 3a-d and 3-chloro-3-(1methylsulphonyl-1H-indol-3-yl)propenal (6) and evaluating their anti-proliferative activity. The structures of the newly synthesized compounds were confirmed by elemental analyses, IR, NMR and mass spectral data. In addition, molecular docking study of the most promising antiproliferative compounds against the active site of carbonic anhydrase IX (PDB ID: 4BCW) theoretically is discussed.
\end{abstract}

Results: Compounds 5c, 7 and 12 revealed potent anti-proliferative effects against A-549 cancer cell line with IC 50 of 44.3 , 17.2 and 38.7 mol/l, respectively compared to the reference drug doxorubicin ( $\mathrm{IC}_{50}$ of $48.8 \mu \mathrm{mol} / \mathrm{l}$ ). While compound $\mathbf{5 c}$ was found to be highly active with $\mathrm{IC}_{50}$ of 5.66 $\mu \mathrm{mol} / \mathrm{l}$ against HCT-116 cancer cell line than doxorubicin (IC 50 of $65.00 \mu \mathrm{mol} / \mathrm{l}$ ).

Conclusion: Further work is recommended to confirm the inhibition of CA IX in a specific bioassay.

Keywords: 1-Methylsulphonyl-3-acetylindole, Heterocycle, Anti-proliferative, Vilsmeier Haack reaction, Molecular docking.

(C) 2016 The Authors. Published by Innovare Academic Sciences Pvt Ltd. This is an open access article under the CC BY license (http://creativecommons.org/licenses/by/4. 0/) DOI: http://dx.doi.org/10.22159/ijpps.2016v8i12.14841

\section{INTRODUCTION}

Classical Vilsmeier-Haack reaction is an efficient method for the formylation of an electron rich aromatic and heterocycles compounds [1]. The reaction of compounds containing acetyl group [2] or its hydrazine [3] with Vilsmeier-Haack reagent is highly versatile and leads to imino alkylations then followed by cyclization to afford aromatic and/or heterocyclic compounds [4-7]. Furthermore, the reaction of compounds containing active methylene group with Vilsmeier-Haack reagent under heating gives the corresponding $\beta$-haloenecarboxaldehyde derivatives $[8,9]$, which are useful precursors in the construction of different heterocyclic systems [8-13]. On the other hand, indoles are one of the most important nitrogen-containing heterocyclic molecules, found extensively in a biological system which plays a vital role in biochemical processes [14]. Furthermore, indole ring constitutes an important template for drug design as they have the unique property of mimicking the structure of peptides and to bind reversibly to enzymes, which provide tremendous opportunities to discover novel drugs with different modes of action $[15,16]$. Moreover, literature revealed that pyrazole, pyrimidine, and triazole are known for their pronounced pharmaceutical activities [17-21]. Human carbonic anhydrase IX (CA IX) is overexpressed in a number of solid tumors and is considered to be a marker for cellular hypoxia that it is not produced in most normal tissues [22]. CA IX contributes to the acidification of the extracellular matrix, which in turn, favors tumor growth and metastasis, therefore, CA IX is considered to be a promising anti-cancer drug target $[23,24]$. Based on the above observations and in continuous of our work [25-29], the present work aimed to synthesize a new series of 1-methylsulphonyl-3indolyl heterocycles via Vilsmeier Haack reaction of 1methylsulphonyl-3-acetylindole and its hydrazone derivatives and then evaluating their anti-proliferative activity. In addition, we attempted to explore the mode of interaction of anti-proliferative compounds with the active site of carbonic anhydrase IX (PDB ID: $4 \mathrm{BCW}$ ) theoretically via molecular docking study.

\section{MATERIALS AND METHODS}

\section{Instruments and reagents}

Melting points were determined on the digital melting point apparatus (Electrothermal 9100, Electrothermal Engineering Ltd, serial No. 8694, Rochford, United Kingdom) and are uncorrected. The microanalytical data were achieved on a Perkin-Elmer 2400 analyzer (Perkin-Elmer, 940 Winter Street, Waltham, Massachusetts 02451 , USA) and were found within $\pm 0.4 \%$ of the theoretical values. IR spectra were recorded on a Perkin-Elmer 1600 Fourier Transform Infrared Spectrophotometer using $\mathrm{KBr}$ discs (PerkinElmer, 940 Winter Street, Waltham, Massachusetts 02451, USA). The NMR spectra were measured with a Bruker Avance digital spectrometer (BRUKER BioSpin GMBH Silberstreifen D-76287 Rheinstetten, Germany) (500 MHz for ${ }^{1} \mathrm{H}$ and $125 \mathrm{MHz}$ for ${ }^{13} \mathrm{C}$ ) in DMSO- $d_{6}$, and the chemical shifts were recorded in $\delta$ ppm relative to TMS as internal standard (all $\mathrm{NH}_{2}$ and $\mathrm{NH}, \mathrm{OH}$ recorded for the compounds were $\mathrm{D}_{2} \mathrm{O}$-exchangeable). Mass spectra (EI) were recorded at $70 \mathrm{eV}$ with JEOL-JMS-AX500 mass spectrometer (JEOL Ltd. 1-2, Musashino 3-chome Akishima, Tokyo 196-8558, Japan). All reagents and solvents were of commercial grade. 1Methylsulphonyl-3-acetylindole was synthesized as reported [30].

\section{Synthesis}

1-(1-(1-Methanesulfony-1H-indol-3-yl) ethylidene)-2-(2-chlorophenyl) hydrazine (2b)

A mixture of 1-methylsulphonyl-3-acetylindole (1) (1g, $4.2 \mathrm{mmol})$, 3-chloro-phenylhydrazine hydrochloride $(0.7 \mathrm{~g}, 4.2 \mathrm{mmol})$ and crystalline sodium acetate $(0.34 \mathrm{~g}, 4.2 \mathrm{mmol})$ was heated under reflux in absolute ethanol $(20 \mathrm{ml})$ for $2 \mathrm{~h}$. After cooling, the reaction 
mixture was poured onto water $(50 \mathrm{ml})$ and the solid that formed was filtered off, washed with water, air dried and crystallized from absolute ethanol. Yield, 82\%; MP: 145-7 ${ }^{\circ} \mathrm{C}$; IR (KBr, $\left.v_{\max }, \mathrm{cm}^{-1}\right) 3180$ $(\mathrm{NH}), 1620(\mathrm{C}=\mathrm{N}), 1575(\mathrm{C}=\mathrm{C}), 1375,1117\left(\mathrm{SO}_{2}\right), 750(\mathrm{Cl}) .{ }^{1} \mathrm{H}$ NMR (500 MHz, DMSO- $\left.d_{6}\right): \delta 2.35\left(\mathrm{~s}, 3 \mathrm{H}, \mathrm{CH}_{3}\right), 3.65\left(\mathrm{~s}, 3 \mathrm{H}, \mathrm{CH}_{3}\right), 7.46-6.79$ (m, 7H, Ar-H), $7.91(\mathrm{~s}, 1 \mathrm{H}, \mathrm{Ph}), 8.54(\mathrm{~s}, 1 \mathrm{H}, \mathrm{H}-2$ indole), $9.52 \mathrm{ppm}(\mathrm{s}$, $1 \mathrm{H}, \mathrm{NH})$; [13]C NMR (125MHz, DMSO- $\left.d_{6}\right): \delta 14.2\left(\mathrm{CH}_{3}\right), 40.8\left(\mathrm{CH}_{3}\right)$, 111.1-147.4 ppm (Ar-C); Anal. $\mathrm{C}_{17} \mathrm{H}_{16} \mathrm{ClN}_{3} \mathrm{O}_{2} \mathrm{~S}$ (361.85): Calcd: C, 56.43; H, 4.46; N, 11.61; Found: C, 56.66; H, 4.42; N, 11.40 .

\section{Synthesis of $1 H$-pyrazole-4-carboxaldehydes 3a-d}

To a solution of compound $2 \mathrm{a}, 2 \mathrm{~b}, 2 \mathrm{c}$ or $2 \mathrm{~d}$ ( $3 \mathrm{mmol}$ ) in $\mathrm{N}, \mathrm{N}$ dimethylformamide $(15 \mathrm{ml})$, phosphorus oxychloride $(1.17 \mathrm{ml}, 10$ mmol) was added drop-wise at $0{ }^{\circ} \mathrm{C}$ while stirring. After complete addition of $\mathrm{POCl}_{3}$, the reaction mixture was left to stir for $15 \mathrm{~h}$ at room temperature and then poured onto ice-water $(20 \mathrm{ml})$. The solid that formed was filtered off, air dried and crystallized from absolute ethanol.

\section{3-(1-Methylsulphonyl-1H-indol-3-yl)-1-phenyl-1H-pyrazole-4- carboxaldehyde (3a)}

Yield, 62\%; MP: $150-2{ }^{\circ} \mathrm{C}$; IR (KBr, $\left.v_{\max }, \mathrm{cm}^{-1}\right) 1715(\mathrm{C}=0), 1618$ $(\mathrm{C}=\mathrm{N}), 1574(\mathrm{C}=\mathrm{C}), 1375,1117\left(\mathrm{SO}_{2}\right) ;{ }^{1} \mathrm{H}$ NMR $\left(500 \mathrm{MHz}\right.$, DMSO- $\left.d_{6}\right)$ : $\delta 3.41\left(\mathrm{~s}, 3 \mathrm{H}, \mathrm{CH}_{3}\right), 7.01-7.89(\mathrm{~m}, 9 \mathrm{H}, \mathrm{Ar}-\mathrm{H}), 8.01(\mathrm{~s}, 1 \mathrm{H}, \mathrm{H}-5$ pyrazole), 8.32 (s, 1H, H-2 indole), $9.91 \mathrm{ppm}(\mathrm{s}, 1 \mathrm{H}, \mathrm{CHO})$; [13]C NMR (125MHz, DMSO- $\left.d_{6}\right): \delta 41.5\left(\mathrm{CH}_{3}\right), 112.9-134.4(\mathrm{Ar}-\mathrm{C}), 193.8$ ppm (C=0); Anal. $\mathrm{C}_{19} \mathrm{H}_{15} \mathrm{~N}_{3} \mathrm{O}_{3} \mathrm{~S}$ (365.41): Calcd: $\mathrm{C}, 62.45 ; \mathrm{H}, 4.14 ; \mathrm{N}$, 11.50; Found: C, 62.55; H, 4.32; N, 11.62 .

\section{3-(1-Methylsulphonyl-1H-indol-3-yl)-1-(2-chlorophenyl)-1H- pyrazole-4-carboxaldehyde (3b)}

Yield, 68\%; MP: $151-3^{\circ} \mathrm{C}$; IR (KBr, $\left.v_{\max }, \mathrm{cm}^{-1}\right) 1702(\mathrm{C}=0), 1618(\mathrm{C}=\mathrm{N})$, 1588 (C=C), 1375, $1117\left(\mathrm{SO}_{2}\right), 750(\mathrm{Cl}) ;{ }^{1} \mathrm{H}$ NMR (500 MHz, DMSO- $\left.d_{6}\right)$ : $\delta 3.36\left(\mathrm{~s}, 3 \mathrm{H}, \mathrm{CH}_{3}\right), 6.80-7.46(\mathrm{~m}, 8 \mathrm{H}, \mathrm{Ar}-\mathrm{H}), 7.90(\mathrm{~s}, 1 \mathrm{H}, \mathrm{H}-5$ pyrazole), 8.52 (s, $1 \mathrm{H}, \mathrm{H}-2$ indole), $9.51 \mathrm{ppm}(\mathrm{s}, 1 \mathrm{H}, \mathrm{CHO})$; [13]C NMR (125MHz, DMSO-d $\left.d_{6}\right): \delta 41.5\left(\mathrm{CH}_{3}\right), 111.1-147.4(\mathrm{Ar}-\mathrm{C}), 191.8 \mathrm{ppm}(\mathrm{C}=0) ; \mathrm{m} / \mathrm{z}$ $\left[\mathrm{M}^{+} / \mathrm{M}^{+}+2\right.$, 399/401, 55/18\%]; Anal. $\mathrm{C}_{19} \mathrm{H}_{14} \mathrm{ClN}_{3} \mathrm{O}_{3} \mathrm{~S}$ (399.85): Calcd: $\mathrm{C}$, 57.07; H, 3.53; N, 10.51; Found: C, 57.22; H, 3.22; N, 10.75.

\section{3-(1-Methylsulphonyl-1H-indol-3-yl)-1-(2, 4-dinitrophenyl)-1H-} pyrazole-4-carboxaldehyde (3c)

Yield, 73\%; MP: 263-5 ${ }^{\circ} \mathrm{C}$; IR (KBr, $\left.v_{\max }, \mathrm{cm}^{-1}\right) 1720$ (C=0), 1602 $(\mathrm{C}=\mathrm{N}), 1577(\mathrm{C}=\mathrm{C}), 1375,1117\left(\mathrm{SO}_{2}\right)$; ${ }^{1} \mathrm{H}$ NMR $\left(500 \mathrm{MHz}\right.$, DMSO- $\left.d_{6}\right)$ : $\delta 3.56\left(\mathrm{~s}, 3 \mathrm{H}, \mathrm{CH}_{3}\right), 7.21-8.51(\mathrm{~m}, 8 \mathrm{H}, \mathrm{Ar}-\mathrm{H}), 8.88(\mathrm{~s}, 1 \mathrm{H}, \mathrm{H}-5$ pyrazole), $11.88 \mathrm{ppm}(\mathrm{s}, 1 \mathrm{H}, \mathrm{CHO})$; [13]C NMR (125MHz, DMSO- $\left.d_{6}\right)$ : $\delta 41.2\left(\mathrm{CH}_{3}\right), 111.9-144.1(\mathrm{Ar}-\mathrm{C}), 153.0 \mathrm{ppm}(\mathrm{C}=0) ; \mathrm{m} / z\left[\mathrm{M}^{+} 455\right.$, 24\%]; Anal. $\mathrm{C}_{19} \mathrm{H}_{13} \mathrm{~N}_{5} \mathrm{O}_{7} \mathrm{~S}$ (455.40): Calcd: C, 50.11; H, 2.88; N, 15.38; Found: C, 50.22; H, 3.00; N, 15.11 .

3-(1-Methylsulphonyl-1H-indol-3-yl)-1-(2, 4, 6-trichlorophenyl) -1H-pyrazole-4-carboxaldehyde (3d)

Yield, 65\%; MP: 70-2 ${ }^{\circ} \mathrm{C}$; IR ( $\left.\mathrm{KBr}, \nu_{\max }, \mathrm{cm}^{-1}\right) 1700(\mathrm{C}=0), 1620(\mathrm{C}=\mathrm{N})$, $1573(\mathrm{C}=\mathrm{C}), 1375,1117\left(\mathrm{SO}_{2}\right), 755(\mathrm{Cl}){ }^{1} \mathrm{H}$ NMR $(500 \mathrm{MHz}, \mathrm{DMSO}-$ $\left.d_{6}\right): \delta 2.90\left(\mathrm{~s}, 3 \mathrm{H}, \mathrm{CH}_{3}\right), 7.43-8.37(\mathrm{~m}, 7 \mathrm{H}, \mathrm{Ar}-\mathrm{H}), 8.60(\mathrm{~s}, 1 \mathrm{H}, \mathrm{H}-5$ pyrazole), $12.35 \mathrm{ppm}(\mathrm{s}, 1 \mathrm{H}, \mathrm{CHO})$; [13]C NMR (125MHz, DMSO- $\left.d_{6}\right)$ : $\delta 41.5\left(\mathrm{CH}_{3}\right), 100.9-143.2(\mathrm{Ar}-\mathrm{C}), 193.7 \mathrm{ppm}(\mathrm{C}=0)$; Anal. $\mathrm{C}_{19} \mathrm{H}_{12} \mathrm{Cl}_{3} \mathrm{~N}_{3} \mathrm{O}_{3} \mathrm{~S}$ (468.74): Calcd: C, 48.68; H, 2.58; N, 8.96; Found: C, 48.42; H, 2.31; N, 9.01.

\section{Synthesis of oximes 4a-d}

A mixture of compound $3 \mathrm{a}, 3 \mathrm{~b}, 3 \mathrm{c}$ or $3 \mathrm{~d}(2 \mathrm{mmol})$, hydroxylamine hydrochloride $(0.14 \mathrm{~g}, 2 \mathrm{mmol})$ and crystalline sodium acetate $(0.16$ g, $2 \mathrm{mmol})$ in absolute ethanol $(20 \mathrm{ml})$ was refluxed for 3-5h. After cooling, the reaction mixture was poured onto water $(20 \mathrm{ml})$ and the solid that formed was filtered off, air dried and crystallized from absolute ethanol.

\section{3-(1-Methylsulphonyl-1H-indol-3-yl)-1-phenyl-1H-pyrazole-4- oxime (4a)}

Yield, $81 \%$; MP: $138-140{ }^{\circ} \mathrm{C}$; IR (KBr, $\left.v_{\max } \mathrm{cm}^{-1}\right) 3400(\mathrm{OH}), 1620$ $(\mathrm{C}=\mathrm{N}), 1573(\mathrm{C}=\mathrm{C}), 1375,1117\left(\mathrm{SO}_{2}\right) ;{ }^{1} \mathrm{H}$ NMR $\left(500 \mathrm{MHz}\right.$, DMSO- $\left.d_{6}\right) \delta$ $3.65\left(\mathrm{~s}, 3 \mathrm{H}, \mathrm{CH}_{3}\right), 7.41-7.91$ (m, 9H, Ar-H), 7.99 (s, 1H, H-5 pyrazole), $8.29(\mathrm{~s}, 1 \mathrm{H}, \mathrm{H}-2$ indole), $8.54(\mathrm{~s}, 1 \mathrm{H}, \mathrm{CH}=\mathrm{N}), 11.18 \mathrm{ppm}(\mathrm{s}, 1 \mathrm{H}, \mathrm{OH})$; [13]C NMR (125MHz, DMSO-d $\left.d_{6}\right): \delta 41.5\left(\mathrm{CH}_{3}\right), 112.7-149.8 \mathrm{ppm}(\mathrm{Ar}-$ C); $m / z$ [M+380, 35\%]; Anal. $\mathrm{C}_{19} \mathrm{H}_{16} \mathrm{~N}_{4} \mathrm{O}_{3} \mathrm{~S}$ (380.42): Calcd: C, 59.99; $\mathrm{H}$, 4.24; N, 14.73; Found: C, 60.11; H, 4.44; N, 14.82.

\section{3-(1-Methylsulphonyl-1H-indol-3-yl)-1-(2-chlorophenyl)-1H- pyrazole-4-oxime (4b)}

Yield, 78\%; MP: $142-4{ }^{\circ} \mathrm{C}$; IR (KBr, $\left.v_{\max } \mathrm{cm}^{-1}\right) 3410(\mathrm{OH}), 1620$ $(\mathrm{C}=\mathrm{N}), 1575(\mathrm{C}=\mathrm{C}), 1375,1117\left(\mathrm{SO}_{2}\right), 750(\mathrm{Cl}) ;{ }^{1} \mathrm{H}$ NMR $(500 \mathrm{MHz}$ DMSO- $\left.d_{6}\right): \delta 3.49\left(\mathrm{~s}, 3 \mathrm{H}, \mathrm{CH}_{3}\right), 7.20-7.46(\mathrm{~m}, 7 \mathrm{H}, \mathrm{Ar}-\mathrm{H}), 7.89(\mathrm{~s}, 1 \mathrm{H}$, $\mathrm{CH}=\mathrm{N}), 8.52(\mathrm{~s}, 1 \mathrm{H}, \mathrm{H}-2$ indole), $9.53(\mathrm{~s}, 1 \mathrm{H}, \mathrm{H}-5$ pyrazole), 11.36 ppm (s, 1H, OH); [13]C NMR (125MHz, DMSO-d $): \delta 41.5\left(\mathrm{CH}_{3}\right)$, 111.7-143.8 ppm (Ar-C); $m / z\left[\mathrm{M}^{+} / \mathrm{M}^{+}+2 ; 414 / 416,21 / 7 \%\right]$; Anal. $\mathrm{C}_{19} \mathrm{H}_{15} \mathrm{ClN}_{4} \mathrm{O}_{3} \mathrm{~S}$ (414.87): Calcd: C, 55.01; H, 3.64; N, 13.50; Found: C, 55.21; H, 3.77; N, 13.64 .

\section{3-(1-Methylsulphonyl-1H-indol-3-yl)-1-(2, 4-dinitrophenyl)-1H-} pyrazole-4-oxime (4c)

Yield, $89 \%$; MP: $323-5{ }^{\circ} \mathrm{C}$; IR (KBr, $\left.v_{\max }, \mathrm{cm}^{-1}\right) 3405(\mathrm{OH}), 1619$ $(\mathrm{C}=\mathrm{N}), 1588(\mathrm{C}=\mathrm{C}), 1375,1117\left(\mathrm{SO}_{2}\right) ;{ }^{1} \mathrm{H}$ NMR $(500 \mathrm{MHz}$, DMSO-d 6 : $\delta 3.37\left(\mathrm{~s}, 3 \mathrm{H}, \mathrm{CH}_{3}\right), 7.20-7.46(\mathrm{~m}, 7 \mathrm{H}, \mathrm{Ar}-\mathrm{H}), 8.02(\mathrm{~s}, 1 \mathrm{H}, \mathrm{CH}=\mathrm{N}), 8.36$ (s, 1H, H-2 indole), 8.83 (s, $1 \mathrm{H}, \mathrm{H}-5$ pyrazole), $11.23 \mathrm{ppm}(\mathrm{s}, 1 \mathrm{H}, \mathrm{OH})$; [13]C NMR (125MHz, DMSO- $\left.d_{6}\right): \delta 45.5\left(\mathrm{CH}_{3}\right), 111.8-153.3 \mathrm{ppm}(\mathrm{Ar}-$ C); $m / z$ [M+470, 25\%]; Anal. $\mathrm{C}_{19} \mathrm{H}_{14} \mathrm{~N}_{6} \mathrm{O}_{7} \mathrm{~S}$ (470.42): Calcd: C, 48.51; $\mathrm{H}$, 3.00; N, 17.87; Found: C, 48.44; H, 2.88; N, 17.54 .

\section{3-(1-Methylsulphonyl-1H-indol-3-yl)-1-(2, 4, 6-trichlorophenyl) -1H-pyrazole-4-oxime (4d)}

Yield, 85\%; MP: 52-4 ${ }^{\circ} \mathrm{C}$; IR (KBr, $\left.v_{\max }, \mathrm{cm}^{-1}\right) 3410(\mathrm{OH}), 1619(\mathrm{C}=\mathrm{N})$, 1588 (C=C), 1375, $1117\left(\mathrm{SO}_{2}\right), 755(\mathrm{Cl}) ;{ }^{1} \mathrm{H}$ NMR $(500 \mathrm{MHz}$, DMSO$\left.d_{6}\right): \delta 3.65\left(\mathrm{~s}, 3 \mathrm{H}, \mathrm{CH}_{3}\right), 7.41-7.49(\mathrm{~m}, 6 \mathrm{H}, \mathrm{Ar}-\mathrm{H}), 8.01(\mathrm{~s}, 1 \mathrm{H}, \mathrm{CH}=\mathrm{N})$, $8.26(\mathrm{~s}, 1 \mathrm{H}, \mathrm{H}-2$ indole), $8.83(\mathrm{~s}, 1 \mathrm{H}, \mathrm{H}-5$ pyrazole), $12.23 \mathrm{ppm}(\mathrm{s}, 1 \mathrm{H}$, $\mathrm{OH}$ ); [13]C NMR (125MHz, DMSO- $\left.d_{6}\right): \delta 41.5\left(\mathrm{CH}_{3}\right), 111.8-153.3 \mathrm{ppm}$ (Ar-C); Anal. $\mathrm{C}_{19} \mathrm{H}_{13} \mathrm{Cl}_{3} \mathrm{~N}_{4} \mathrm{O}_{3} \mathrm{~S}$ (483.76): Calcd: C, 47.17; H, 2.71; N, 11.58; Found: C, 47.32; H, 2.55; N, 11.42 .

\section{Synthesis of $1 \mathrm{H}$-pyrazole-4-carbonitriles 5a-d}

A solution of compound $4 \mathrm{a}, 4 \mathrm{~b}, 4 \mathrm{c}$ or $4 \mathrm{~d}(2 \mathrm{mmol})$ in acetic anhydride $(15 \mathrm{ml})$ was heated gently for $2 \mathrm{~h}$. After cooling, the reaction mixture was poured onto ice-water and the solid that formed was filtered off, washed with water, air dried and crystallized from absolute ethanol.

\section{3-(1-Methylsulphonyl-1H-indol-3-yl)-1-phenyl-1H-pyrazole-4- carbonitrile (5a)}

Yield, 90\%; MP: 210-212 ${ }^{\circ} \mathrm{C}$; IR ( $\left.\mathrm{KBr}, v_{\max }, \mathrm{cm}^{-1}\right) 2197$ (CN), 1618 $(\mathrm{C}=\mathrm{N}), 1575(\mathrm{C}=\mathrm{C}), 1385,1175\left(\mathrm{SO}_{2}-\mathrm{N}\right) ;{ }^{1} \mathrm{H}$ NMR $(500 \mathrm{MHz}, \mathrm{DMSO}-$ $\left.d_{6}\right): \delta 2.94\left(\mathrm{~s}, 3 \mathrm{H}, \mathrm{CH}_{3}\right), 6.99-7.68(\mathrm{~m}, 9 \mathrm{H}, \mathrm{Ar}-\mathrm{H}), 8.23(\mathrm{~s}, 1 \mathrm{H}, \mathrm{H}-2$ indole), $8.71 \mathrm{ppm}(\mathrm{s}, 1 \mathrm{H}, \mathrm{H}-5$ pyrazole); $\mathrm{m} / \mathrm{z}$ [M+362, 23\%]; Anal. $\mathrm{C}_{19} \mathrm{H}_{14} \mathrm{~N}_{4} \mathrm{O}_{2} \mathrm{~S}$ (362.41): Calcd: C, 62.97; H, 3.89; N, 15.46; Found: $\mathrm{C}$, 62.88; H, 4.00; N, 15.66 .

\section{3-(1-Methylsulphonyl-1H-indol-3-yl)-1-(2-chlorophenyl)-1H- pyrazole-4-carbonitrile (5b)}

Yield, 88\%; MP: $235 \mathrm{dec}^{\circ} \mathrm{C}$; IR (KBr, $\left.\nu_{\max }, \mathrm{cm}^{-1}\right) 2195$ (CN), 1618 (C=N), 1602 (C=C), 1365, 1135 ( $\left.\mathrm{SO}_{2}-\mathrm{N}\right), 752$ (C-Cl); ${ }^{\mathrm{H}} \mathrm{H}$ NMR (500 MHz, DMSO$\left.d_{6}\right): \delta 3.26\left(\mathrm{~s}, 3 \mathrm{H}, \mathrm{CH}_{3}\right), 7.16-7.78(\mathrm{~m}, 8 \mathrm{H}, \mathrm{Ar}-\mathrm{H}), 8.23(\mathrm{~s}, 1 \mathrm{H}, \mathrm{H}-2$ indole), 8.65 ppm (s, $1 \mathrm{H}, \mathrm{H}-5$ pyrazole); Anal. $\mathrm{C}_{19} \mathrm{H}_{13} \mathrm{ClN}_{4} \mathrm{O}_{2} \mathrm{~S}$ (396.85): Calcd: C, 57.50; H, 3.30; N, 14.12; Found: C, 57.66; H, 3.22; N, 14.01.

\section{3-(1-Methylsulphonyl-1H-indol-3-yl)-1-(2,4-dinitrophenyl)-1H-} pyrazole-4-carbonitrile (5c)

Yield, 81\%; MP: $270 \mathrm{dec}{ }^{\circ} \mathrm{C}$; IR (KBr, $\left.v_{\max }, \mathrm{cm}^{-1}\right) 2205(\mathrm{CN}), 1620$ $(\mathrm{C}=\mathrm{N}), 1533(\mathrm{C}=\mathrm{C}), 1365,1142\left(\mathrm{SO}_{2}-\mathrm{N}\right) ;{ }^{1} \mathrm{H}$ NMR $(500 \mathrm{MHz}, \mathrm{DMSO}-$ $\left.d_{6}\right): \delta 3.31\left(\mathrm{~s}, 3 \mathrm{H}, \mathrm{CH}_{3}\right), 7.27-7.82(\mathrm{~m}, 7 \mathrm{H}, \mathrm{Ar}-\mathrm{H}), 8.45(\mathrm{~s}, 1 \mathrm{H}, \mathrm{H}-2$ indole), $8.81 \mathrm{ppm}\left(\mathrm{s}, 1 \mathrm{H}, \mathrm{H}-5\right.$ pyrazole); $\mathrm{m} / z\left[\mathrm{M}^{+} / \mathrm{M}^{+}+2 ; 452 / 454\right.$, 27/9\%]; Anal. $\mathrm{C}_{19} \mathrm{H}_{12} \mathrm{~N}_{6} \mathrm{O}_{6} \mathrm{~S}$ (452.40): Calcd: C, 50.44; H, 2.67; N, 18.58; Found: C, 50.24; H, 2.77; N, 18.42.

3-(1-Methylsulphonyl-1H-indol-3-yl)-1-(2, 4, 6-trichlorophenyl) -1H-pyrazole-4-carbonitrile (5d)

Yield, 79\%; MP: $110-2^{\circ} \mathrm{C}$; IR ( $\left.\mathrm{KBr}, \nu_{\max }, \mathrm{cm}^{-1}\right) 2197$ (CN), 1617 (C=N), 1585 (C=C), 1376, $1138\left(\mathrm{SO}_{2}-\mathrm{N}\right), 757$ (C-Cl); ${ }^{1} \mathrm{H}$ NMR (500 MHz, DMSO- 
$\left.d_{6}\right): \delta 3.11\left(\mathrm{~s}, 3 \mathrm{H}, \mathrm{CH}_{3}\right), 7.01-7.63(\mathrm{~m}, 6 \mathrm{H}, \mathrm{Ar}-\mathrm{H}), 7.99(\mathrm{~s}, 1 \mathrm{H}, \mathrm{H}-5$ pyrazole), $8.23 \mathrm{ppm}\left(\mathrm{s}, 1 \mathrm{H}, \mathrm{H}-2\right.$ indole); Anal. $\mathrm{C}_{19} \mathrm{H}_{11} \mathrm{Cl}_{3} \mathrm{~N}_{4} \mathrm{O}_{2} \mathrm{~S}$ (465.74): Calcd: C, 49.00; H, 2.38; N, 12.03; Found: C, 49.22; H, 2.55; N, 11.89 .

\section{3-Chloro-3-(1-methylsulphonyl-1H-indol-3-yl) propenal (6)}

To a solution of compound $1(1 \mathrm{~g}, 4.2 \mathrm{mmol})$ in $N$, $N$-dimethyl formamide (15 ml), phosphorus oxychloride $(1.17 \mathrm{ml}, 12 \mathrm{mmol})$ was added drop-wise at $0{ }^{\circ} \mathrm{C}$ while stirring. After complete addition of $\mathrm{POCl}_{3}$, the reaction mixture was warmed to room temperature and then heated at $60{ }^{\circ} \mathrm{C}$ for $3 \mathrm{~h}$. After cooling, the reaction mixture was poured onto crushed ice and then neutralized with $10 \%$ aqueous sodium hydroxide solution. The precipitate that formed was filtered off, washed with water, air dried and crystallized methanol. Yield, 60\%; MP: $110-2{ }^{\circ} \mathrm{C}$; IR (KBr, $\left.v_{\max }, \mathrm{cm}^{-1}\right) 1720$ (C=0), 1600 (C=C), 1375, $1117\left(\mathrm{SO}_{2}\right), 748(\mathrm{Cl}) ;{ }^{1} \mathrm{H}$ NMR $\left(500 \mathrm{MHz}\right.$, DMSO-d $\left.\mathrm{d}_{6}\right)$ : v $3.65(\mathrm{~s}$, $\left.3 \mathrm{H}, \mathrm{CH}_{3}\right), 7.41-7.49(\mathrm{~m}, 4 \mathrm{H}, \mathrm{Ar}-\mathrm{H}), 7.91(\mathrm{~s}, 1 \mathrm{H}, \mathrm{CH}=\mathrm{C}), 8.29$ (s, $1 \mathrm{H}, \mathrm{H}-2$ indole), $8.53 \mathrm{ppm}(\mathrm{s}, 1 \mathrm{H}, \mathrm{CHO})$; [13]C NMR (125MHz, DMSO- $\left.d_{6}\right): \delta$ $27.7\left(\mathrm{CH}_{3}\right), 112.9-134.4$ (Ar-C), $193.85 \mathrm{ppm}(\mathrm{C}=0) ; \mathrm{m} / z[\mathrm{M}+283$, 26\%]; Anal. $\mathrm{C}_{12} \mathrm{H}_{10} \mathrm{ClNO}_{3} \mathrm{~S}$ (283.73): Calcd: C, 50.80; H, 3.55; N, 4.94; Found: C, 50.64; H, 3.33; N, 5.01.

\section{5-(1-Methylsulphonyl-1H-indol-3-yl)-1H-pyrazole (7)}

A mixture of compound 6 (0.57 g, $2 \mathrm{mmol})$ and hydrazine hydrate $99 \%(0.1 \mathrm{~g}, 2 \mathrm{mmol})$ in absolute ethanol $(15 \mathrm{ml})$ containing few drops of glacial acetic acid was refluxed for $3 \mathrm{~h}$. After cooling, the reaction mixture was poured onto water $(20 \mathrm{ml})$ and the solid that formed was filtered off, air dried and crystallized from methanol. Yield, 67\%; MP: 233-5 ${ }^{\circ} \mathrm{C}$; IR (KBr, $\left.v_{\max }, \mathrm{cm}^{-1}\right) 3180(\mathrm{NH}), 1620(\mathrm{NH})$, 1578 (C=C), 1375, $1117\left(\mathrm{SO}_{2}\right)$; ${ }^{1} \mathrm{H}$ NMR (500 MHz, DMSO-d $): \delta 2.54$ $(\mathrm{s}, 1 \mathrm{H}, \mathrm{NH}), 3.64\left(\mathrm{~s}, 3 \mathrm{H}, \mathrm{CH}_{3}\right), 7.46-7.50(\mathrm{~m}, 4 \mathrm{H}, \mathrm{Ar}-\mathrm{H}), 7.94(\mathrm{~d}, 1 \mathrm{H}, \mathrm{H}-$ 4 pyrazole), 8.29 (s, $1 \mathrm{H}, \mathrm{H}-2$ indole), $8.58 \mathrm{ppm}(\mathrm{d}, 1 \mathrm{H}, \mathrm{H}-5$ pyrazole); [13]C NMR (125MHz, DMSO- $\left.d_{6}\right): \delta 41.16\left(\mathrm{CH}_{3}\right), 112.8-157.0 \mathrm{ppm}$ (Ar-C); Anal. $\mathrm{C}_{12} \mathrm{H}_{11} \mathrm{~N}_{3} \mathrm{O}_{2} \mathrm{~S}$ (261.30): Calcd: C, 55.16; H, 4.24; N, 16.08; Found: C, 55.23; H, 4.44; N, 16.22.

\section{1-(5-(1-Methylsulphonyl-1H-indol-3-yl)-1H-pyrazol-1-yl) ethanone (8)}

To a solution of compound $6(0.57 \mathrm{~g}, 2 \mathrm{mmol})$ in a mixture of $(10 \mathrm{ml})$ acetic anhydride and glacial acetic acid (2:1), was added hydrazine hydrate $99 \%(0.1 \mathrm{ml}, 2 \mathrm{mmol})$. The reaction mixture was refluxed for $4 \mathrm{~h}$. After cooling, the reaction mixture was poured onto icewater $(50 \mathrm{ml})$, and the solid that formed was filtered off, air dried and crystallized from aqueous ethanol. Yield, $61 \%$; MP: $75-7^{\circ} \mathrm{C}$; IR $\left(\mathrm{KBr}, v_{\max }, \mathrm{cm}^{-1}\right) 1688(\mathrm{C}=0), 1612(\mathrm{C}=\mathrm{N}), 1554(\mathrm{C}=\mathrm{C}), 1375,1117$ $\left(\mathrm{SO}_{2}\right) ;{ }^{1} \mathrm{H}$ NMR (500 MHz, DMSO-d $): \delta 1.84\left(\mathrm{~s}, 3 \mathrm{H}, \mathrm{CH}_{3}\right), 3.64(\mathrm{~s}, 3 \mathrm{H}$, $\left.\mathrm{CH}_{3}\right), 7.52-7.97(\mathrm{~m}, 4 \mathrm{H}, \mathrm{Ar}-\mathrm{H}), 8.21(\mathrm{~d}, 1 \mathrm{H}, \mathrm{H}-4$ pyrazole $), 8.38(\mathrm{~d}, 1 \mathrm{H}$, $\mathrm{H}-5$ pyrazole), $8.49 \mathrm{ppm}$ (s, $1 \mathrm{H}, \mathrm{H}-2$ indole); [13]C NMR (125MHz, DMSO- $\left.d_{6}\right): \delta 20.3,41.16\left(2 \mathrm{CH}_{3}\right), 112.8-157.0$ (Ar-C), $168.0 \mathrm{ppm}$ (C=0); $m / z[\mathrm{M}+303,42 \%]$; Anal. $\mathrm{C}_{14} \mathrm{H}_{13} \mathrm{~N}_{3} \mathrm{O}_{3} \mathrm{~S}$ (303.34): Calcd: $\mathrm{C}$, 55.43; H, 4.32; N, 13.85; Found: C, 55.22; H, 4.11; N, 15.00 .

\section{5-(1-Methylsulphonyl-1H-indol-3-yl)-1-phenyl-1H-pyrazole (9)}

A mixture of compound 6 (0.57 g, $2 \mathrm{mmol})$ and phenyl hydrazine $(0.22 \mathrm{~g}, 2 \mathrm{mmol})$ in absolute ethanol $(15 \mathrm{ml})$ containing few drops of glacial acetic acid was refluxed for $3 \mathrm{~h}$. After cooling, the reaction mixture was poured onto water $(20 \mathrm{ml})$ and the solid that formed was filtered off, air dried and crystallized from methanol. Yield, 35\%; MP: 65-7 ${ }^{\circ} \mathrm{C}$; IR ( $\left.\mathrm{KBr}, v_{\max }, \mathrm{cm}^{-1}\right) 1618(\mathrm{C}=\mathrm{N}), 1563(\mathrm{C}=\mathrm{C}), 1376,1135$ $\left(\mathrm{SO}_{2}-\mathrm{N}\right) ;{ }^{1} \mathrm{H}$ NMR (500 MHz, DMSO-d 6 ): $\delta 2.85\left(\mathrm{~s}, 1 \mathrm{H}, \mathrm{CH}_{3}\right), 7.06-7.72$ $(\mathrm{m}, 10 \mathrm{H}, \mathrm{Ar}-\mathrm{H}, \mathrm{H}-3$ pyrazole), $6.56(\mathrm{~s}, 1 \mathrm{H}, \mathrm{H}-4$ pyrazole), $8.32 \mathrm{ppm}(\mathrm{s}$, $1 \mathrm{H}, \mathrm{H}-2$ indole); Anal. $\mathrm{C}_{18} \mathrm{H}_{15} \mathrm{~N}_{3} \mathrm{O}_{2} \mathrm{~S}$ (337.40): Calcd: C, 64.08; $\mathrm{H}, 4.48$; N, 12.45; Found: C, 64.22; H, 5.00; N, 12.66.

\section{5-(1-Methylsulphonyl-1H-indol-3-yl)-1-benzyl-1H-pyrazole (10)}

A mixture of compound 6 (0.57 g, $2 \mathrm{mmol})$, benzylhydrazine dihydrochloride $(0.39 \mathrm{~g}, 2 \mathrm{mmol})$ and sodium acetate $(0.3 \mathrm{~g}, 4 \mathrm{mmol})$ in absolute ethanol $(15 \mathrm{ml})$ was refluxed for $3 \mathrm{~h}$. After cooling, the reaction mixture was poured onto water $(20 \mathrm{ml})$ and the solid that formed was filtered off, air dried and crystallized from absolute ethanol. Yield, 46\%; MP: $237 \mathrm{dec}{ }^{\circ} \mathrm{C}$; IR (KBr, $\left.v_{\max }, \mathrm{cm}^{-1}\right) 1620(\mathrm{C}=\mathrm{N})$, $1601(\mathrm{C}=\mathrm{C}), 1381,1142\left(\mathrm{SO}_{2}-\mathrm{N}\right) ;{ }^{1} \mathrm{H}$ NMR $\left(500 \mathrm{MHz}, \mathrm{DMSO}-d_{6}\right): \delta$ $3.25\left(\mathrm{~s}, 1 \mathrm{H}, \mathrm{CH}_{3}\right), 5.56\left(\mathrm{~s}, 2 \mathrm{H}, \mathrm{CH}_{2}-\mathrm{N}\right)$ 7.19-7.76 (m, 10H, Ar-H, H-3 pyrazole), $6.63(\mathrm{~d}, 1 \mathrm{H}, \mathrm{H}-4$ pyrazole), $8.25 \mathrm{ppm}(\mathrm{s}, 1 \mathrm{H}, \mathrm{H}-2$ indole); $m / z$ [M+351, 40\%]; Anal. $\mathrm{C}_{19} \mathrm{H}_{17} \mathrm{~N}_{3} \mathrm{O}_{2} \mathrm{~S}$ (351.42): Calcd: C, 64.94; $\mathrm{H}$, 4.88; N, 11.96; Found: C, 65.00; H, 5.00; N, 12.00.

\section{6-(1-Methylsulphonyl-1H-indol-3-yl) pyrimidin-2(1H)-thione (11)}

A mixture of compound $6(0.57 \mathrm{~g}, 2 \mathrm{mmol})$ and thiourea $(0.15 \mathrm{~g}, 2$ $\mathrm{mmol})$ in absolute ethanol $(15 \mathrm{ml})$ containing glacial acetic acid $(0.5$ $\mathrm{ml}$ ) was refluxed for $2 \mathrm{~h}$. After cooling, the reaction mixture was poured onto ice-water $(50 \mathrm{ml})$ and the solid that formed was filtered off, air dried and crystallized from absolute ethanol. Yield, 36\%; MP: 124-6 ${ }^{\circ} \mathrm{C}$; IR (KBr, v $\left.v_{\max }, \mathrm{cm}^{-1}\right) 3210(\mathrm{NH}), 1618(\mathrm{C}=\mathrm{N}), 1577$ (C=C), 1240 (C=S), 1375, $1117\left(\mathrm{SO}_{2}\right) ;{ }^{1} \mathrm{H}$ NMR (500 MHz, DMSO- $\left.d_{6}\right): \delta 3.64$ (s, 3H, $\left.\mathrm{CH}_{3}\right), 7.44-7.49(\mathrm{~m}, 4 \mathrm{H}, \mathrm{Ar}-\mathrm{H}), 7.91(\mathrm{~d}, 1 \mathrm{H}, \mathrm{CH}$ pyrimidine), $8.29(\mathrm{~d}, 1 \mathrm{H}, \mathrm{CH}$ pyrimidine), $8.53(\mathrm{~s}, 1 \mathrm{H}, \mathrm{H}-2$ indole $), 11.58 \mathrm{ppm}(\mathrm{s}$, $1 \mathrm{H}, \mathrm{NH})$; [13]C NMR (125MHz, DMSO- $\left.d_{6}\right): \delta 41.5\left(\mathrm{CH}_{3}\right), 112.9-143.4$ (Ar-C), 193.8 ppm (C=S); m/z [M+305, 35\%]; Anal. $\mathrm{C}_{13} \mathrm{H}_{11} \mathrm{~N}_{3} \mathrm{O}_{2} \mathrm{~S}_{2}$ (305.38): Calcd: C, 51.13; H, 3.63; N, 13.76; Found: C, 51.22; H, 3.77; N, 13.55 .

6-(1-Methylsulphonyl-1H-indol-3-yl) pyrimidin-2(1H)-one (12)

A mixture of compound $6(0.57 \mathrm{~g}, 2 \mathrm{mmol})$ and urea $(0.12 \mathrm{~g}, 2 \mathrm{mmol})$ in absolute ethanol $(15 \mathrm{ml})$ containing glacial acetic acid $(0.5 \mathrm{ml})$ was refluxed for $2 \mathrm{~h}$. After cooling, the reaction mixture was poured onto ice-water $(50 \mathrm{ml})$ and the solid that formed was filtered off, air dried and crystallized from absolute ethanol. Yield, 32\%; MP: 84-6 ${ }^{\circ} \mathrm{C}$; IR (KBr, $\left.v_{\max }, \mathrm{cm}^{-1}\right) 3218(\mathrm{NH}), 1654$ (C=0), 1618 (C=N), 1574 $(\mathrm{C}=\mathrm{C}), 1375,1117\left(\mathrm{SO}_{2}\right) ;{ }^{1} \mathrm{H}$ NMR $\left(500 \mathrm{MHz}, \mathrm{DMSO}-d_{6}\right): \delta 3.64(\mathrm{~s}, 3 \mathrm{H}$, $\mathrm{CH}_{3}$ ), 7.41-7.49 (m, 4H, Ar-H), 7.92 (d, 1H, CH pyrimidine), 8.29 (d, $1 \mathrm{H}, \mathrm{CH}$ pyrimidine), 8.53 (s, $1 \mathrm{H}, \mathrm{H}-2$ indole), $9.58 \mathrm{ppm}(\mathrm{s}, 1 \mathrm{H}, \mathrm{NH})$; [13]C NMR (125MHz, DMSO-d6): $\delta 41.5\left(\mathrm{CH}_{3}\right), 111.9-156.4(\mathrm{Ar}-\mathrm{C})$, $175.8 \mathrm{ppm}(\mathrm{C}=0) ; \mathrm{m} / z$ [M+289, 22\%]; Anal. $\mathrm{C}_{13} \mathrm{H}_{11} \mathrm{~N}_{3} \mathrm{O}_{3} \mathrm{~S}$ (289.31): Calcd: C, 53.97; H, 3.83; N, 14.52; Found: C, 53.72; H, 4.01; N, 14.33 .

\section{4-(1-Methylsulphonyl-1H-indol-3-yl) pyrimidin-2-amine (13)}

A mixture of compound $6(2.83 \mathrm{~g}, 10 \mathrm{mmol})$, guanidine hydrochloride $(0.96 \mathrm{~g}, 10 \mathrm{mmol})$ and crystalline sodium acetate $(0.82 \mathrm{~g}, 10 \mathrm{mmol})$ in absolute ethanol $(15 \mathrm{ml})$ was refluxed for $2-3 \mathrm{~h}$. The solid that formed on hot was filtered off, air dried and crystallized from absolute ethanol. Yield, 30\%; MP: $143-5{ }^{\circ} \mathrm{C}$; IR $\left(\mathrm{KBr}, v_{\max }, \mathrm{cm}^{-1}\right) 3260,2168\left(\mathrm{NH}_{2}\right), 1620(\mathrm{C}=\mathrm{N}), 1581(\mathrm{C}=\mathrm{C}), 1375$, $1117\left(\mathrm{SO}_{2}\right) ;{ }^{1} \mathrm{H}$ NMR $\left(500 \mathrm{MHz}\right.$, DMSO-d $\left.d_{6}\right): \delta 2.58\left(\mathrm{~s}, 3 \mathrm{H}, \mathrm{CH}_{3}\right), 7.42-$ $7.48(\mathrm{~m}, 4 \mathrm{H}, \mathrm{Ar}-\mathrm{H}), 7.90$ (d, 1H, CH pyrimidine), 8.29 (d, $1 \mathrm{H}, \mathrm{CH}$ pyrimidine), 8.53 (s, $1 \mathrm{H}, \mathrm{H}-2$ indole), $11.65 \mathrm{ppm}\left(\mathrm{s}, 2 \mathrm{H}, \mathrm{NH}_{2}\right.$ ); [13]C NMR (125MHz, DMSO-d 6 ): $\delta 41.5\left(\mathrm{CH}_{3}\right), 111.1-147.4 \mathrm{ppm}(\mathrm{Ar}-\mathrm{C})$; $m / z$ [M+288, 16\%]; Anal. $\mathrm{C}_{13} \mathrm{H}_{12} \mathrm{~N}_{4} \mathrm{O}_{2} \mathrm{~S}$ (288.32): Calcd: C, 54.15; $\mathrm{H}$, 4.20; N, 19.43; Found: C, 54.23; H, 4.00; N, 19.22.

\section{Synthesis of benzamides $14 a, b$}

A mixture of compound 13 ( $0.57 \mathrm{~g}, 2 \mathrm{mmol})$ and benzoyl chloride or 4-chlorobenzoyl chloride $(2 \mathrm{mmol})$ in dry dioxane containing triethylamine $(1 \mathrm{ml})$ was refluxed for $3 \mathrm{~h}$. After cooling, the reaction mixture was poured onto water $(30 \mathrm{ml})$ and the solid that formed was filtered off, air dried and crystallized from absolute ethanol.

\section{N-(4-(1-Methylsulphonyl-1H-indol-3-yl)pyrimidin-2-yl) benzamide (14a)}

Yield, 25\%; MP: 99-101 ${ }^{\circ} \mathrm{C}$; IR (KBr, $\left.v_{\max }, \mathrm{cm}^{-1}\right) 3200(\mathrm{NH}), 1664$ $(\mathrm{C}=0), 1601(\mathrm{C}=\mathrm{N}), 1588(\mathrm{C}=\mathrm{C}), 1375,1117\left(\mathrm{SO}_{2}\right) ;{ }^{1} \mathrm{H}$ NMR $(500$ MHz, DMSO-d6): $\delta 3.35\left(\mathrm{~s}, 2 \mathrm{H}, \mathrm{CH}_{3}\right), 7.58-7.72(\mathrm{~m}, 9 \mathrm{H}, \mathrm{Ar}-\mathrm{H}, \mathrm{CH}$ pyrimidine), $7.90(\mathrm{~d}, 1 \mathrm{H}, \mathrm{CH}$ pyrimidine), $8.00(\mathrm{~s}, 1 \mathrm{H}, \mathrm{H}-2$ indole), $11.99 \mathrm{ppm}(\mathrm{s}, 1 \mathrm{H}, \mathrm{NH})$; [13]C NMR (125MHz, DMSO- $\left.d_{6}\right): \delta 40.0$ $\left(\mathrm{CH}_{3}\right)$, 127.7-148.9 (Ar-C), 166.7 ppm (C=O); Anal. $\mathrm{C}_{20} \mathrm{H}_{16} \mathrm{~N}_{4} \mathrm{O}_{3} \mathrm{~S}$ (392.43): Calcd: C, 61.21; H, 4.11; N, 14.28; Found: C, 61.00; H, 4.21; $\mathrm{N}, 14.35$.

\section{N-(4-(1-Methylsulphonyl-1H-indol-3-yl)pyrimidin-2-yl)-4- chlorobenzamide (14b)}

Yield, 22\%; MP: $133-135{ }^{\circ} \mathrm{C}$; IR ( $\left.\mathrm{KBr}, v_{\max }, \mathrm{cm}^{-1}\right) 3265(\mathrm{NH}), 1685$ $(\mathrm{C}=0), 1616(\mathrm{C}=\mathrm{N}), 1565(\mathrm{C}=\mathrm{C}), 1365,1124\left(\mathrm{SO}_{2}-\mathrm{N}\right), 752(\mathrm{C}-\mathrm{Cl}) ;{ }^{1} \mathrm{H}$ NMR (500 MHz, DMSO- $\left.d_{6}\right): \delta 3.14\left(\mathrm{~s}, 3 \mathrm{H}, \mathrm{CH}_{3}\right), 7.15-7.78(\mathrm{~m}, 8 \mathrm{H}, \mathrm{Ar}-$ $\mathrm{H}, \mathrm{CH}$ pyrimidine), 7.95 (d, 1H, CH pyrimidine), $8.16(\mathrm{~s}, 1 \mathrm{H}, \mathrm{H}-2$ indole), $9.27 \mathrm{ppm}(\mathrm{s}, 1 \mathrm{H}, \mathrm{NH}) ; \mathrm{m} / z\left[\mathrm{M}^{+} / \mathrm{M}^{+}+2 ; 426 / 428,42 / 14 \%\right]$; Anal. $\mathrm{C}_{20} \mathrm{H}_{15} \mathrm{ClN}_{4} \mathrm{O}_{3} \mathrm{~S}$ (426.88): Calcd: C, 56.27; H, 3.54; N, 13.12; Found: C, 56.44; H, 5.32; N, 13.01 . 


\section{Synthesis of sulphonamides 15a, b}

A mixture of compound 13 ( $0.57 \mathrm{~g}, 2 \mathrm{mmol})$ and benzene sulphonyl chloride or 4-bromobenzenesulphonyl chloride $(2 \mathrm{mmol})$ in dry dioxane containing triethylamine $(1 \mathrm{ml})$ was refluxed for $3 \mathrm{~h}$. After cooling, the reaction mixture was poured onto water $(30 \mathrm{ml})$ and the solid that formed was filtered off, air dried and crystallized from absolute ethanol.

\section{N-(4-(1-Methylsulphonyl-1H-indol-3-yl) pyrimidin-2-yl)-2- benzenesulphonamide (15a)}

Yield, 32\%; MP: $168-171{ }^{\circ} \mathrm{C}$; IR (KBr, $\left.v_{\max }, \mathrm{cm}^{-1}\right) 3165(\mathrm{NH}), 1672$ $(\mathrm{C}=0), 1618(\mathrm{C}=\mathrm{N}), 1575(\mathrm{C}=\mathrm{C}), 1385,1133\left(\mathrm{SO}_{2}-\mathrm{N}\right) ;{ }^{1} \mathrm{H}$ NMR $(500$ MHz, DMSO-d $): \delta 2.95\left(\mathrm{~s}, 3 \mathrm{H}, \mathrm{CH}_{3}\right), 7.06-7.77(\mathrm{~m}, 10 \mathrm{H}, \mathrm{Ar}-\mathrm{H}, \mathrm{CH}$ pyrimidine), $7.86(\mathrm{~d}, 1 \mathrm{H}, \mathrm{CH}$ pyrimidine), $8.20(\mathrm{~s}, 1 \mathrm{H}, \mathrm{H}-2$ indole), $8.85 \mathrm{ppm}(\mathrm{s}, 1 \mathrm{H}, \mathrm{NH}) ; \mathrm{m} / z[\mathrm{M}+428,12 \%]$; Anal. $\mathrm{C}_{19} \mathrm{H}_{16} \mathrm{~N}_{4} \mathrm{O}_{4} \mathrm{~S}_{2}$ (428.48): Calcd: C, 53.26; H, 3.76; N, 13.08; Found: C, 53.44; H, 3.66; N, 12.98 .

N-(4-(1-Methylsulphonyl-1H-indol-3-yl) pyrimidin-2-yl)-2-(4bromobenzene) sulphonamide (15b)

Yield, 26\%; MP: 98-100 ${ }^{\circ} \mathrm{C}$; IR (KBr, $\left.v_{\max } \mathrm{cm}^{-1}\right) 3224(\mathrm{NH}), 1692$ $(\mathrm{C}=0), 1618(\mathrm{C}=\mathrm{N}), 1553(\mathrm{C}=\mathrm{C}), 1361,1142\left(\mathrm{SO}_{2}-\mathrm{N}\right), 755(\mathrm{C}-\mathrm{Br}) ;{ }^{1} \mathrm{H}$ NMR (500 MHz, DMSO- $\left.d_{6}\right): \delta 3.31\left(\mathrm{~s}, 3 \mathrm{H}, \mathrm{CH}_{3}\right), 7.21-7.83(\mathrm{~m}, 9 \mathrm{H}, \mathrm{Ar}-$ $\mathrm{H}, \mathrm{CH}$ pyrimidine), $7.90(\mathrm{~d}, 1 \mathrm{H}, \mathrm{CH}$ pyrimidine), $8.32(\mathrm{~s}, 1 \mathrm{H}, \mathrm{H}-2$ indole), $9.62 \mathrm{ppm}(\mathrm{s}, 1 \mathrm{H}, \mathrm{NH}) ; \mathrm{m} / z\left[\mathrm{M}^{+} / \mathrm{M}^{+}+2 ; 507 / 509,21 / 21 \%\right]$; Anal. $\mathrm{C}_{19} \mathrm{H}_{15} \mathrm{BrN}_{4} \mathrm{O}_{4} \mathrm{~S}_{2}$ (507.38): Calcd: C, 44.98; H, 2.98; N, 11.04; Found: C, 45.01; H, 3.01; N, 10.99 .

\section{N-4-(1-Methylsulphonyl-1H-indol-3-yl) pyrimidin-2-yl) acetamide} (16)

A solution of compound $13(0.57 \mathrm{~g}, 2 \mathrm{mmol})$ in a mixture of acetic anhydride and glacial acetic acid $(2: 1,10 \mathrm{ml})$ was heated under reflux for $10 \mathrm{~h}$. After cooling, the reaction mixture was poured onto ice-water $(50 \mathrm{ml})$, and the solid that formed was filtered off, air dried and crystallized from aqueous ethanol. Yield, 31\%; MP: 226-8 ${ }^{\circ} \mathrm{C}$; IR (KBr, v $\left.v_{\max } \mathrm{cm}^{-1}\right) 3206(\mathrm{NH}), 1677$ (C=0), 1617 (C=N), 1601 $(\mathrm{C}=\mathrm{C}), 1375,1127\left(\mathrm{SO}_{2}-\mathrm{N}\right)$; ${ }^{1} \mathrm{H}$ NMR $\left(500 \mathrm{MHz}, \mathrm{DMSO}-d_{6}\right): \delta 2.01(\mathrm{~s}$, $\left.3 \mathrm{H}, \mathrm{CH}_{3}\right), 3.37\left(\mathrm{~s}, 3 \mathrm{H}, \mathrm{CH}_{3}\right), 7.35-7.54(\mathrm{~m}, 4 \mathrm{H}, \mathrm{Ar}-\mathrm{H}), 7.61,7.92(2 \mathrm{~d}$, $2 \mathrm{H}, 2 \mathrm{CH}$ pyrimidine), 8.21 (s, $1 \mathrm{H}, \mathrm{H}-2$ indole), $8.75 \mathrm{ppm}(\mathrm{s}, 1 \mathrm{H}, \mathrm{NH})$; $m / z$ [M+330, 34\%]; Anal. $\mathrm{C}_{15} \mathrm{H}_{14} \mathrm{~N}_{4} \mathrm{O}_{3} \mathrm{~S}$ (330.36): Calcd: C, 54.53; $\mathrm{H}$, 4.27; N, 16.96; Found: C, 54.32; H, 4.09; N, 17.01.

\section{4-(1-Methylsulphonyl-1H-indol-3-yl)-2-azidopyrimidine (17)}

To a cooled solution of compound $13(5.76 \mathrm{~g}, 20 \mathrm{mmol})$ in concentrated sulfuric acid (5 ml) and ice (15 g), cooled solution of sodium nitrite $(1.73 \mathrm{~g}, 25 \mathrm{mmol})$ in ice-water $(10 \mathrm{ml})$ was added dropwise while stirring at $0-5^{\circ} \mathrm{C}$ and keeping at this temperature for $10 \mathrm{~min}$. To this solution of diazonium salt, sodium azide $(1.3 \mathrm{~g}, 20$ $\mathrm{mmol}$ ) in ice-water $(5 \mathrm{ml})$ was added drop-wise while stirring. The solution was left at room temperature for $15 \mathrm{~min}$ then the azide was extracted by diethyl ether. Ether was evaporated in vacuo and azide was used without subsequent cleaning. 2-Azidopyrimidine derivative (17) was identified by chromatography-mass spectrometry since it decomposed slowly during the preparation of the analyzed sample. Also, it used in the reaction immediately after its formation because of its instability: Yield, $22 \%$; MP: $98-100{ }^{\circ} \mathrm{C}$; $m / z\left[\mathrm{M}^{+} 314,52 \%\right]$.

5-Methyl-1-(4-(1-methylsulphonyl-1H-indol-3-yl) pyrimidin-2yl)-1H-1,2,3-triazole-4-carboxylic acid (18)

To a solution of sodium $(0.23 \mathrm{~g}, 10 \mathrm{mmol})$ in absolute methanol $(20$ $\mathrm{ml})$ was added ethyl acetoacetate $(1.34 \mathrm{~g}, 10 \mathrm{mmol})$ and compound $17(3.14 \mathrm{~g}, 10 \mathrm{mmol})$ dropwise while stirring and cooling in an icebath. The reaction mixture was kept in ice water bath for $30 \mathrm{~min}$ and then gradually heated under reflux for $1 \mathrm{~h}$. After cooling, the reaction mixture was neutralized using diluted hydrochloric acid (1:1). The solid that formed was filtered off, washed with water, air-dried and crystallized from methanol. Yield, $24 \%$; MP: $120-2{ }^{\circ} \mathrm{C}$; IR $\left(\mathrm{KBr}, v_{\max }\right.$, $\left.\mathrm{cm}^{-1}\right) 3445(\mathrm{OH}), 1668(\mathrm{C}=0), 1620(\mathrm{C}=\mathrm{N}), 1569(\mathrm{C}=\mathrm{C}), 1365,1135$ $\left(\mathrm{SO}_{2}-\mathrm{N}\right) ;{ }^{1} \mathrm{H}$ NMR $\left(500 \mathrm{MHz}, \mathrm{DMSO}-d_{6}\right): \delta 1.61\left(\mathrm{~s}, 3 \mathrm{H}, \mathrm{CH}_{3}\right), 3.27(\mathrm{~s}$, $\left.3 \mathrm{H}, \mathrm{CH}_{3}\right), 7.26-7.46(\mathrm{~m}, 4 \mathrm{H}, \mathrm{Ar}-\mathrm{H}), 7.62,8.18(2 \mathrm{~d}, 2 \mathrm{H}, 2 \mathrm{CH}$ pyrimidine), 8.42 (s, $1 \mathrm{H}, \mathrm{H}-2$ indole), $11.50 \mathrm{ppm}(\mathrm{s}, 1 \mathrm{H}, \mathrm{OH}) ; \mathrm{m} / \mathrm{z}$
[M+398, 25\%]; Anal. $\mathrm{C}_{17} \mathrm{H}_{14} \mathrm{~N}_{6} \mathrm{O}_{4} \mathrm{~S}$ (398.40): Calcd: C, 51.25; H, 3.54; N, 21.09; Found: C, 51.44; H, 3.42; N, 21.22.

3-(4-(1-Methylsulphonyl-1H-indol-3-yl) 1,2,3-triazol-4-amine (19)

pyrimidin-2-yl)-1H-

To a solution of sodium $(0.23 \mathrm{~g}, 10 \mathrm{mmol})$ in absolute methanol $(20$ $\mathrm{ml})$, acetonitrile $(0.41 \mathrm{~g}, 10 \mathrm{mmol})$ was added. To this solution, compound 17 (3.14 g, $10 \mathrm{mmol})$ in dry methanol $(5 \mathrm{ml})$ was added dropwise and left to stir for $24 \mathrm{~h}$. The resulting precipitate was filtered off, washed with water, air-dried and crystallized from methanol. Yield, 21\%; MP: $237-5{ }^{\circ} \mathrm{C}$; IR (KBr, $\left.v_{\max }, \mathrm{cm}^{-1}\right) 3365\left(\mathrm{NH}_{2}\right)$, $1616(\mathrm{C}=\mathrm{N}), 1535(\mathrm{C}=\mathrm{C}), 1355,1124\left(\mathrm{SO}_{2}-\mathrm{N}\right) ;{ }^{1} \mathrm{H}$ NMR $(500 \mathrm{MHz}$, DMSO- $\left.d_{6}\right): \quad \delta 2.84\left(\mathrm{~s}, 3 \mathrm{H}, \mathrm{CH}_{3}\right), 7.21-7.65(\mathrm{~m}, 5 \mathrm{H}, \mathrm{Ar}-\mathrm{H}, \mathrm{CH}$ pyrimidine), $7.92(\mathrm{~s}, 1 \mathrm{H}, \mathrm{CH}$ triazole), $8.15(\mathrm{~d}, 1 \mathrm{H}, \mathrm{CH}$ pyrimidine), 8.31 (s, $1 \mathrm{H}, \mathrm{H}-2$ indole), $11.50 \mathrm{ppm}\left(\mathrm{s}, 2 \mathrm{H}, \mathrm{NH}_{2}\right) ; \mathrm{m} / \mathrm{z}[\mathrm{M}+355,12 \%]$; Anal. $\mathrm{C}_{15} \mathrm{H}_{13} \mathrm{~N}_{7} \mathrm{O}_{2} \mathrm{~S}$ (355.37): Calcd: C, 50.70; H, 3.69; N, 27.59; Found: C, 50.82; H, 3.54; N, 27.44.

\section{N-(4-Nitrobenzylidene)-4-(1-methylsulphonyl-1H-indol-3-yl)} pyrimidin-2-amine (20)

A mixture of compound $13(1 \mathrm{~g}, 3 \mathrm{mmol})$ and 4-nitrobenzaldehyde $(0.45 \mathrm{~g}, 3 \mathrm{mmol})$ in absolute ethanol $(20 \mathrm{ml})$ containing glacial acetic acid $(1 \mathrm{ml})$ was refluxed for $3 \mathrm{~h}$. After cooling, the reaction mixture was poured onto water $(30 \mathrm{ml})$ and the solid that formed was filtered off, air dried and crystallized from absolute ethanol. Yield, 57\%; MP: 85-7 ${ }^{\circ} \mathrm{C}$; IR ( $\left.\mathrm{KBr}, v_{\max ,} \mathrm{cm}^{-1}\right) 1620(\mathrm{C}=\mathrm{N}), 1596(\mathrm{C}=\mathrm{C}), 1345,1133\left(\mathrm{SO}_{2}-\right.$ $\mathrm{N}) ;{ }^{1} \mathrm{H}$ NMR (500 MHz, DMSO- $\left.d_{6}\right): \delta 3.29\left(\mathrm{~s}, 3 \mathrm{H}, \mathrm{CH}_{3}\right), 7.17-8.18(\mathrm{~m}$, $10 \mathrm{H}, \mathrm{Ar}-\mathrm{H}, 2 \mathrm{CH}$ pyrimidine), $8.26(\mathrm{~s}, 1 \mathrm{H}, \mathrm{H}-2$ indole), $8.45 \mathrm{ppm}(\mathrm{s}, 1 \mathrm{H}$, $\mathrm{CH}=\mathrm{N}) ; \mathrm{m} / z$ [M+421, 1\%]; Anal. $\mathrm{C}_{20} \mathrm{H}_{15} \mathrm{~N}_{5} \mathrm{O}_{4} \mathrm{~S}$ (421.43): Calcd: C, 57.00; H, 3.59; N, 16.62; Found: C, 57.22; H, 3.72; N, 16.55 .

3-(4-(1-Methylsulphonyl-1H-indol-3-yl) pyrimidin-2-yl)-2-(4nitrophenyl) thiazolidin-4-one (21)

To a stirred solution of compound $20(0.8 \mathrm{~g}, 2 \mathrm{mmol})$ in dry dioxane $(25 \mathrm{ml})$ was added thioglycolic acid $(0.024 \mathrm{~g}, 3 \mathrm{mmol})$. After stirring for $4 \mathrm{~h}$, anhydrous sodium sulfate $(5 \mathrm{~g})$ was added and then refluxed for another $6 \mathrm{~h}$. The reaction mixture was filtered while hot. After cooling, the solid that formed was filtered off, washed with water, air-dried and crystallized from dioxane. Yield, 33\%; MP: 175-177 ${ }^{\circ} \mathrm{C}$; IR (KBr, $\left.v_{\max }, \mathrm{cm}^{-1}\right) 1702(\mathrm{C}=0), 1622(\mathrm{C}=\mathrm{N}), 1587(\mathrm{C}=\mathrm{C}), 1356,1133$ ( $\left.\mathrm{SO}_{2}-\mathrm{N}\right) ;{ }^{1} \mathrm{H}$ NMR (500 MHz, DMSO- $\left.d_{6}\right): \delta 2.96\left(\mathrm{~s}, 3 \mathrm{H}, \mathrm{CH}_{3}\right), 3.72(\mathrm{~s}$, $2 \mathrm{H}, \mathrm{H}-4$ thiazolidinone), $5.92(\mathrm{~s}, 1 \mathrm{H}, \mathrm{H}-2$ thiazolidinone), 7.07-8.19 (m, 10H, Ar-H, 2CH pyrimidine), $8.32 \mathrm{ppm}(\mathrm{s}, 1 \mathrm{H}, \mathrm{H}-2$ indole); $\mathrm{m} / \mathrm{z}$ [M+495, 5\%]; Anal. $\mathrm{C}_{22} \mathrm{H}_{17} \mathrm{~N}_{5} \mathrm{O}_{5} \mathrm{~S}_{2}$ (495.53): Calcd: C, 53.32; H, 3.46; N, 14.13; Found: C, 53.12; H, 3.26; N, 14.44 .

2-(4-(1-Methylsulphonyl-1H-indol-3-yl) pyrimidin-2-yl)amino)2-(4-nitrophenyl) acetonitrile (22)

To a solution of compound $20(0.8 \mathrm{~g}, 2 \mathrm{mmol})$ in acetic acid $(15 \mathrm{ml})$, sodium cyanide $(0.11 \mathrm{~g}, 2 \mathrm{mmol})$ was added and the reaction mixture was refluxed for $4 \mathrm{~h}$. After cooling, the reaction mixture was poured onto water $(30 \mathrm{ml})$ and the solid that formed was filtered off, air dried and crystallized from absolute ethanol. Yield, 63\%; MP: 116-118 ${ }^{\circ} \mathrm{C}$; IR (KBr, $\left.v_{\max }, \mathrm{cm}^{-1}\right) 3212(\mathrm{NH}), 2197(\mathrm{CN}), 1702(\mathrm{C}=0)$, $\left.1618(\mathrm{C}=\mathrm{N}), 1565(\mathrm{C}=\mathrm{C}), 1341,1117\left(\mathrm{SO}_{2}-\mathrm{N}\right) ; \mathrm{m} / z \quad \mathrm{M}+448,2 \%\right]$; Anal. $\mathrm{C}_{21} \mathrm{H}_{16} \mathrm{~N}_{6} \mathrm{O}_{4} \mathrm{~S}$ (448.45): Calcd: C, 56.24; $\mathrm{H}, 3.60 ; \mathrm{N}, 18.74$; Found: C, $56.35 ; \mathrm{H}, 3.76$; N, 18.66 .

2-(4-(1-Methylsulphonyl-1H-indol-3-yl) pyrimidin-2-yl)amino)2-(4-nitrophenyl) acetic acid (23)

A solution of compounds $22(4.48 \mathrm{~g}, 10 \mathrm{mmol})$ in sulfuric acid $(30 \mathrm{ml}$, $50 \%$ ) was heated at reflux for $10 \mathrm{~h}$. After cooling, the dark reaction mixture was poured onto cold water $(20 \mathrm{ml})$ and then neutralized with ammonia solution (25\%). The precipitate that formed was filtered off, washed with water, air-dried, and crystallized from aqueous acetic acid. Yield, 20\%; MP: $214 \mathrm{dec}^{\circ}{ }^{\circ} \mathrm{C}$; IR (KBr, $\left.\nu_{\max }, \mathrm{cm}^{-1}\right) 3435(\mathrm{OH}), 3242$ $(\mathrm{NH}), 1705(\mathrm{C}=0) 1620(\mathrm{C}=\mathrm{N}), 1592(\mathrm{C}=\mathrm{C}), 1363,1122\left(\mathrm{SO}_{2}-\mathrm{N}\right) ;{ }^{1} \mathrm{H}$ NMR (500 MHz, DMSO-d6): $\delta 3.01\left(\mathrm{~s}, 3 \mathrm{H}, \mathrm{CH}_{3}\right), 4.78(\mathrm{~s}, 1 \mathrm{H}, \mathrm{CH}), 5.96(\mathrm{~s}$, $1 \mathrm{H}, \mathrm{NH}), 6.56(\mathrm{~s}, 1 \mathrm{H}, \mathrm{OH}) 7.15-7.87(\mathrm{~m}, 9 \mathrm{H}, \mathrm{Ar}-\mathrm{H}, \mathrm{CH}$ pyrimidine), 8.06 (d, $1 \mathrm{H}, \mathrm{CH}$ pyrimidine), $8.32 \mathrm{ppm}(\mathrm{s}, 1 \mathrm{H}, \mathrm{H}-2$ indole); $\mathrm{m} / \mathrm{z}[\mathrm{M}+467$, 15\%]; Anal. $\mathrm{C}_{21} \mathrm{H}_{17} \mathrm{~N}_{5} \mathrm{O}_{6} \mathrm{~S}$ (467.45): Calcd: C, 53.96; $\mathrm{H}, 3.67 ; \mathrm{N}, 14.98$; Found: C, 54.01; H, 3.45; N, 15.00 . 


\section{Biological assays}

\section{Cell culture}

A-549 (human lung cancer), MCF7 (human breast cancer) and HCT-116 (human colon cancer) cell lines were obtained from Karolinska Institute, Stockholm, Sweden. All cells were maintained in RPMI 1640 medium, except for A-549 cancer cells which were maintained in DMEM medium (Lonza Biowahittkar, Belgium). All the media were supplemented with $1 \%$ antibiotic-antimycotic mixture $(10,000 \mathrm{U} / \mathrm{ml}$ potassium penicillin, $10,000 \mu \mathrm{g} / \mathrm{ml}$ streptomycin sulfate, $25 \mu \mathrm{g} / \mathrm{ml}$ amphotericin $\mathrm{B}$ and $1 \% \mathrm{~L}$ glutamine (Biowest, USA).

\section{MTT cytotoxicity assay}

Cell viability was investigated using MTT [3-(4, 5-dimethylthiazol-2yl)-2, 5-diphenyltetrazolium bromide] (Bio Basic Canada Inc., Canada) assay [31]. This reaction depends on the mitochondrial reduction of yellow MTT into purple formazan. All the preceding steps were carried out in sterile laminar air flow cabinet Biosafety class II level (Baker, SG403INT; Sanford, ME, USA).

All incubations were done at $37{ }^{\circ} \mathrm{C}$ in $5 \% \mathrm{CO}_{2}$ incubator in the humidified atmosphere (Sheldon, TC2323; Cornelius, OR, USA). Cells were seeded into 96-well microtiter plastic plates at the concentration of (104 cells/well) and allowed to adhere for $24 \mathrm{~h}$. The medium was aspirated and fresh medium (without serum) was added to the cells with various concentrations of the test compounds $(100,50,25,12.5,6.25,3.12,1.56$ and $0.78 \mu \mathrm{g} / \mathrm{ml}$ in DMSO) and incubated for $48 \mathrm{~h}$.

The medium was aspirated and $40 \mu \mathrm{l}$ MTT salt $(2.5 \mu \mathrm{g} / \mathrm{ml})$ was added to each well and incubated for a further $4 \mathrm{~h}$. To stop the reaction and dissolve any formed formazan crystals, $200 \mu \mathrm{l}$ of $10 \%$ sodium dodecyl sulfate (SDS) were added to each well and incubated overnight at $37{ }^{\circ} \mathrm{C}$. The amount of formazan product was measured at $595 \mathrm{~nm}$ with a reference wavelength of $620 \mathrm{~nm}$ as a background using a microplate reader (Bio-Rad Laboratories, model 3350, USA). For the untreated cells (negative control), the medium was added instead of the test compounds. A positive control Adrinamycin ${ }^{\circledR} \quad$ (doxorubicin) $\quad(\mathrm{Mr}=579.9) \quad$ (Pharmacia India Pvt Ltd, Gurgaon, Haryana 122001, India) was used as a known cytotoxic natural agent giving 100\% inhibition. Dimethyl sulfoxide (DMSO) was the vehicle used for dissolution of the testing compound and its final concentration on the cells was less than $0.2 \%$. IC 50 was calculated for the samples and negative control (cells with vehicle) by the probit analysis using a simple ttest (SPSS statistical analysis software package/version 11.0, SPSS Inc., (IL), Chicago, USA).

\section{Molecular docking study}

Docking study of the most anti-proliferative compounds 5c, 7 and 12 was performed by the molecular operating environment (MOE) 2008.10 releases of chemical computing group, Montreal, Canada (http://www.chemcomp.com). The program operated on intel(R) core(TM) i3-32100 CPU@3.10G $3.10 \mathrm{GHz}$ processor, 4.00 GB of RAM, Microsoft Windows 7 professional.

The protein crystal structure of carbonic anhydrase IX (PDB ID: $4 \mathrm{BCW})$ in complex with (E)-2-(5-bromo-2-hydroxyphenyl) ethene sulphonic acid (TU0) was downloaded from protein data bank (http://www.rcsb.org/pdb) (PDB ID: 4BCW) [32].

The protein crystal structure was prepared for docking via removing of water molecules, addition and removal of polar hydrogen atoms then isolation of the active pocket. CA IX active site contains Zn (II) in coordination with His94, His96, His119 beside Thr199, Thr200, Leu198, Val121, Val143, Trp209, Gln92, Gln97, Gln106, Phe131, Pro201, Pro202

The co-crystalline ligand was re-docked in the active pocket to insure the docking method was efficient and the active pocket was saved as moe file to be used for docking simulation of the selected compounds (ligands).

The structure of the selected compounds (ligands) for docking was drawn in ChemDraw Ultra 10.0 (Chem Office package) and saved as mol. Before the molecular docking, the preparation steps must be done as follow, a) converting the 2D structure of ligands to their 3D form, b) addition and removing of polar hydrogen atoms; c) energy minimized using the MMFF94x force field until an RMSD (Root-mean-square deviation) of atomic position gradient of 0.01 $\mathrm{Kcal} / \mathrm{mol} / \AA$ was reached and saved as moe. MMFF94x was reported as the efficient force field for minimizing ligand-protein complexes [33].

The docking algorithm was done by MOE-DOCK default. It uses flexible, a rigid technique for posing the molecule inside the cavity. All rotatable bonds of ligands are allowed to undergo free rotation to explore the conformational space inside the rigid receptor binding site. The docking scores were expressed in negative energy terms, the lower the binding free energy, the better the binding affinity [34], and the ligand interactions (hydrogen bonding and hydrophobic interaction) with carbonic anhydrase IX was determined.

\section{RESULTS AND DISCUSSION}

\section{Chemistry}

Schemes 1-3 outline the synthetic pathway to obtain the new target compounds. The required starting materials were prepared via the condensation of 1-methylsulphonyl-3-acetylindole (1) with some hydrazines, namely phenylhydrazine, 2,4-dinitrophenylhydrazine, 2,4,6-trichlorophenylhydrazine under heating in acetic acid to give the corresponding hydrazones $2 \mathrm{a}, 2 \mathrm{c}$, and $2 \mathrm{~d}$.

While hydrazone $2 \mathrm{~b}$, was prepared via the reaction of 1 with 2chlorophenylhydrazine hydrochloride under heating in ethanol and in the presence of crystalline sodium acetate (Scheme 1). Compounds $2 \mathrm{a}, 2 \mathrm{c}$, and $2 \mathrm{~d}$ are previously reported [35], and the newly compound $2 \mathrm{~b}$ was confirmed by its correct elemental analysis and spectral data.

Vilsmeier-Haack formulation of the latter hydrazones 2a-d using 2.5 equivalent moles of Vilsmeier reagent (DMF/POCl 3 ) at $0-5{ }^{\circ} \mathrm{C}$ for $15 \mathrm{~h}$ performed double addition of the reagent on methyl group to afford ultimately after hydrolysis the cyclized substituted phenyl-1H-pyrazole-4-carboxaldehydes 3a-d, respectively (Scheme 1). IR spectra of 3a-d showed strong absorption bands around $1702-1720 \mathrm{~cm}^{-1}$ for the keto of aldehydic groups. Their ${ }^{1} \mathrm{H}$ NMR spectra lacked the singlet signals of the methyl group of hydrazones 3a-d and revealed new singlet signals at $\delta 9.51-12.35 \mathrm{ppm}$ for $\mathrm{CHO}$ proton besides the singlet signals at $\delta$ 7.90-8.88 ppm for $\mathrm{H}-5$ of pyrazole.

Condensation of compounds 3a-d with hydroxylamine hydrochloride under heating in ethanol and in the presence of crystalline sodium acetate led to the formation of the corresponding pyrazole-4-oxime derivatives $4 a-d$, respectively (Scheme 1). IR spectra of 4a-d showed strong absorption bands ranging from 3400 to $3410 \mathrm{~cm}^{-1}$ related to $\mathrm{OH}$ groups besides the characteristic absorption bands of $\mathrm{C}=\mathrm{N}$ around $1620 \mathrm{~cm}^{-1}$, and their ${ }^{1} \mathrm{H} \mathrm{NMR}$ spectra showed singlet signal of the $\mathrm{OH}$ group ranging from 11.18 to $12.23 \mathrm{ppm}$.

Heating of the latter oximes in acetic anhydride yielded the corresponding pyrazole-4-carbonitrile derivatives $5 \mathrm{a}$-d, respectively in good yields ranging from 79 to $90 \%$ (Scheme 1). IR spectra of $5 \mathrm{a}-\mathrm{d}$ showed strong absorption bands ranging from 2195 to $2205 \mathrm{~cm}^{-1}$ for the $\mathrm{CN}$ groups, and their ${ }^{1} \mathrm{H}$ NMR spectra lack the singlet signals of $\mathrm{OH}$ group besides the other signals of the compounds which located at their positions. 
<smiles>CS(=O)(=O)n1cc(/C(Cl)=C/C=O)c2ccccc21</smiles>

6<smiles>CCCCCCCOCCO</smiles>
$60{ }^{0} \mathrm{C}, 1 \mathrm{~h}$<smiles>CC(=O)c1cn(S(C)(=O)=O)c2ccccc12</smiles>

1<smiles>CS(=O)(=O)n1cc(-c2nn(-c3ccccc3)cc2C=N)c2ccccc21</smiles>

4

$\mathrm{Ac}_{2} \mathrm{O}$ reflux<smiles></smiles><smiles>C/C(=N/Nc1ccccc1)c1cn(S(C)(=O)=O)c2ccc(N)cc12</smiles>

2

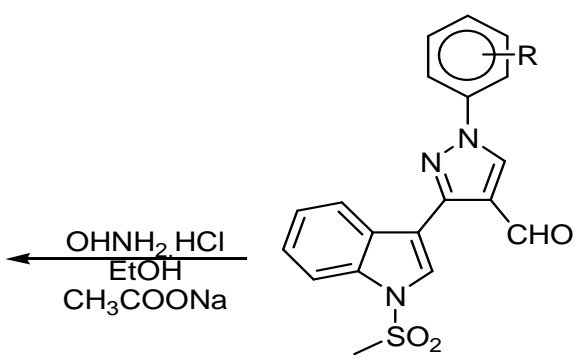

3

$\mathrm{a}=\mathrm{H}$

$b=2-\mathrm{Cl}$

$\mathbf{c}=2,4-\left(\mathrm{NO}_{2}\right)_{2}$

$\mathbf{d}=2,4,6-(\mathrm{Cl})_{3}$

Scheme 1: Syntheses of vilsmeier-haack products 3a-d and 6

On the other hand, the reaction of 1-methylsulphonyl-3-acetylindole (1) with 2.5 equivalent moles of Vilsmeier reagent (DMF/ $\mathrm{POCl}_{3}$ ) under heating at $60{ }^{\circ} \mathrm{C}$ for $1 \mathrm{~h}$ (Vilsmeier-Haack Arnold reaction) led to the formation of 3-chloro-3-(1-methylsulphonyl-1H-indol-3-yl) propenal (6) in $30 \%$ yield (Scheme 1). Its ${ }^{1} \mathrm{H}$ NMR revealed singlet signal at $7.91 \mathrm{ppm}$ due to $\mathrm{CH}=\mathrm{C}$, besides singlet signal at $8.53 \mathrm{ppm}$ issued for $\mathrm{CHO}$.

Although, the very low yield of compound 6 , but it seems to has some interest due to the presence of $\alpha, \beta$-bifunctional chloro and aldehydic group. The reaction of compound 6 with hydrazine hydrate in absolute ethanol and in the presence of few drops of glacial acetic acid afforded 5-(1-methylsulphonyl- $1 H$-indol-3-yl)$1 H$-pyrazole (7). Whereas, the reaction of compound 6 with hydrazine hydrate under reflux in a mixture of acetic anhydride and glacial acetic acid (2:1) afforded the corresponding $\mathrm{N}$ acetylpyrazole derivative 8 (Scheme 2). Additionally, the reaction of compound 6 with phenylhydrazine gave $\mathrm{N}$ phenylpyrazole derivative 9 . Also, the reaction of compound 6 with benzylhydrazine hydrochloride in the presence of anhydrous sodium acetate gave $N$-benzylpyrazole derivative 10 (Scheme 2).

Furthermore, the reaction of compound 6 with thiourea or urea in ethanol in the presence of a glacial acetic acid as a catalyst gave 6-(1methylsulphonyl-1H-indol-3-yl) pyrimidin-2(1H)-thione (11) and 6-(1methylsulphonyl- $1 H$-indol-3-yl)pyrimidin-2(1H)-one (12), respectively. Moreover, the reaction of compound 6 with guanidine hydrochloride in ethanol and in the presence of crystalline sodium acetate yielded 4-(1-methylsulphonyl-1H-indol-3-yl) pyrimidin-2-amine (Scheme 2).

The reaction of compound 13 with benzoyl chloride, 4chlorobenzoyl chloride, benzene sulphonyl chloride or 4bromo-benzenesulphonyl chloride in dry dioxane and in the presence of triethylamine as a base afforded $N$-benzamide derivatives $14 \mathrm{a}, \mathrm{b}$, and $N$-sulphonamide derivatives $15 \mathrm{a}, \mathrm{b}$, respectively (Scheme 3). 


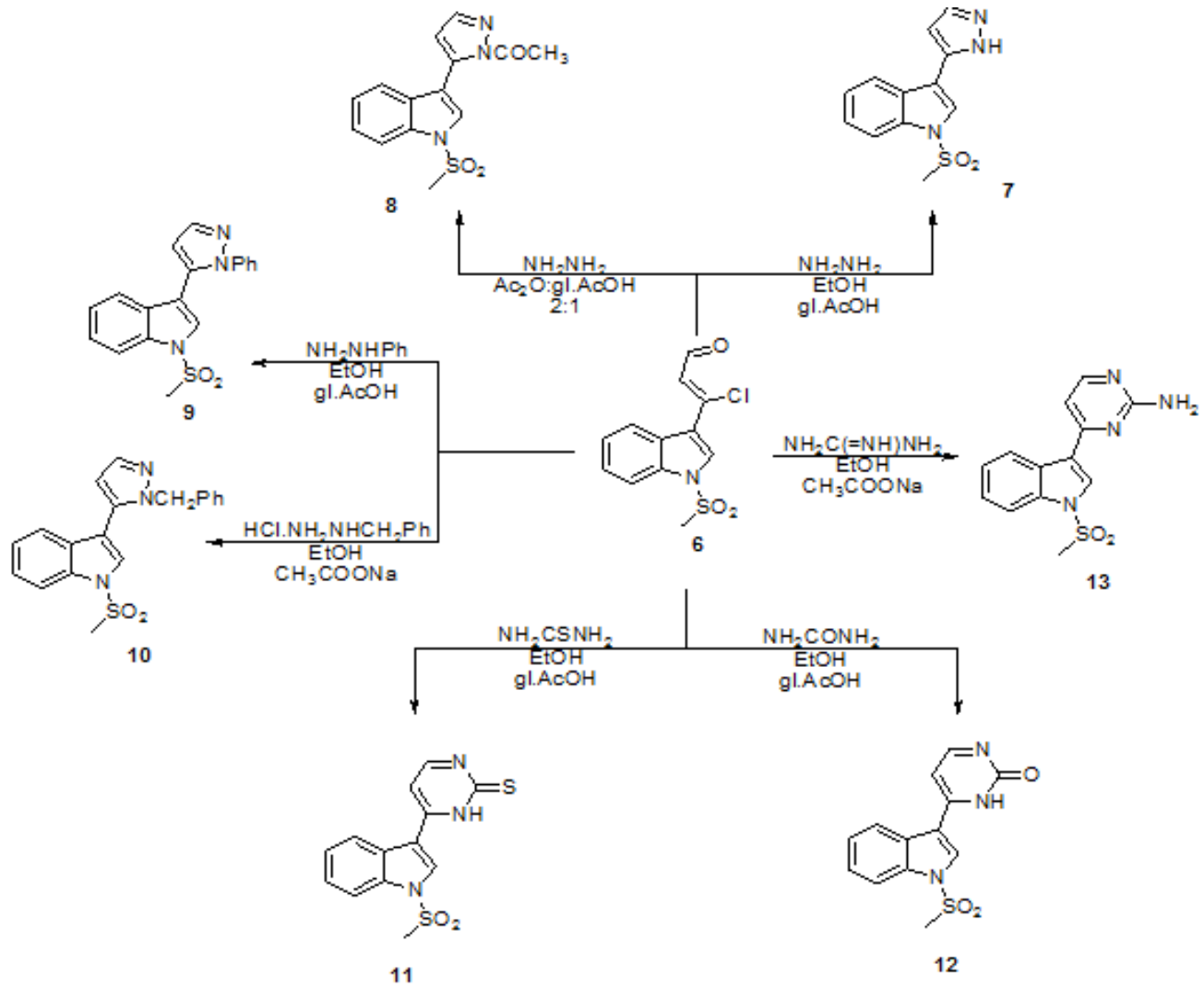

Scheme 2: Syntheses of pyrazoles 7-10 and pyrimidine 11-13 derivatives

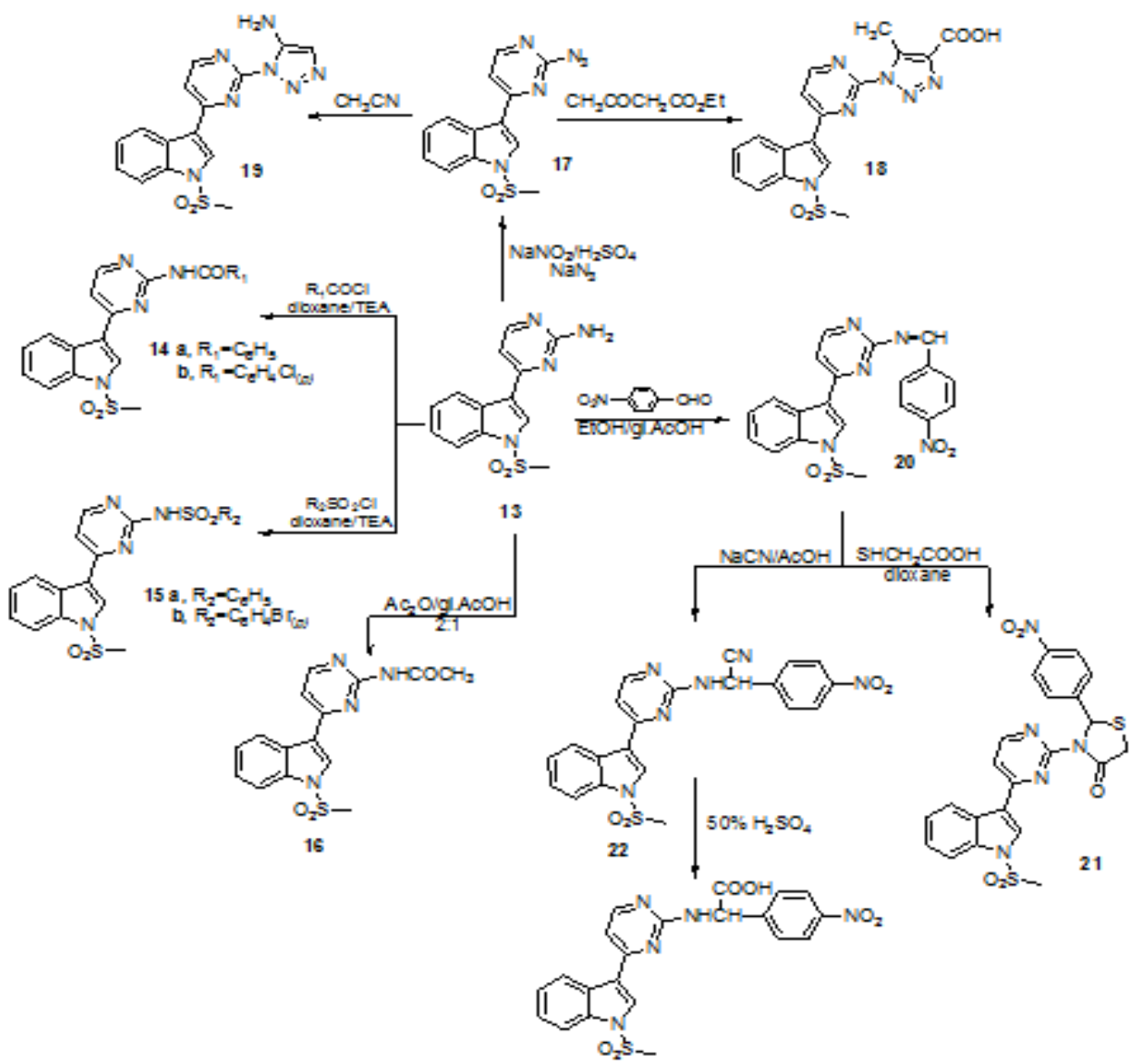

Scheme 3: Syntheses of compounds 14-23 
Acetylation of compound 13 using a mixture of acetic anhydride and glacial acetic acid $(2: 1)$ led to the formation of N-4-(1-methylsulphonyl-1H-indol-3-yl) pyrimidin-2-yl)acetamide (16) (Scheme 3).

Diazotization of compound 13 with concentrated sulphuric acid and sodium nitrite at $0-5{ }^{\circ} \mathrm{C}$ yielded the corresponding diazonium salt which, under reaction with sodium azide yielded 2-azidopyrimidine derivative 17 (Scheme 3). It was previously reported that organic azide undergoes base catalyze condensation reaction with activated methylenic compounds give 1,2,3-triazoles moiety [36]. In the present work and under the above-mentioned conditions, the newly 2-azidopyrimidine derivative (17) was allowed to react with ethyl acetoacetate in the presence of sodium methoxide to give the corresponding 5-methyl-1-(4-(1-methylsulphonyl-1 $H$-indol-3-yl) pyrimidin-2-yl)-1H-1,2,3-triazole-4-carboxylic acid (18) (Scheme 3). Furthermore, the reaction of the compound (17) with acetonitrile in the presence of sodium methoxide led to the formation of 3-(4-(1methylsulphonyl-1H-indol-3-yl) pyrimidin-2-yl)-1H-1,2,3-triazol-4amine (19) (Scheme 3).

Acid catalyzes the reaction of compound 13 with 4-nitrobenzaldehyde in absolute ethanol led to the formation of the corresponding Schiff's base 20 (Scheme 3). Cyclocondensation of compound 20 with thioglycolic acid in the presence of anhydrous sodium sulfate yielded thiazolidin-4-one derivative (21). On the other hand, addition of sodium cyanide to Schiff's base 20 in acetic acid afforded 2-(4-(1-methylsulphonyl-1H-indol-3-yl)pyrimidin-2yl)amino)-2-(4-nitrophenyl)acetonitrile (22), which on hydrolysis with dilute sulfuric acid gave 2-(4-(1-methylsulphonyl-1H-indol-3- yl) pyrimidin-2-yl)amino)-2-(4-nitrophenyl) acetic acid (23) (Scheme 3).

\section{Cytotoxic activity}

Some new compounds numerically labeled with 3a-d, 5a-d, and 712 were preliminarily screened for their in vitro antiproliferative activity against human lung cancer (A-549), human colon cancer (HCT-116) and human breast cancer (MCF-7) cell lines at a concentration of $100 \mu \mathrm{g} / \mathrm{ml}$ (table 1 ). Compounds 3c, 3d, 5c, 7, 9 and 12 showed potent to moderate antiproliferative activity against A-549 cancer cell line of $70,72,95,92,75$ and $92 \%$, respectively. Whereas compounds 5c, 7 and 8 showed, activity against HCT-116 cancer cell line of 83, 92 and 70\%, respectively. Only compounds $5 \mathrm{c}$ and 7 showed moderate activity against MCF7 of 81 and $73 \%$, respectively.

Compounds that showed anti-proliferative activity higher than $80 \%$ at a concentration of $100 \mu \mathrm{g} / \mathrm{ml} \mathrm{(5c,7} \mathrm{and} \mathrm{12)} \mathrm{were} \mathrm{used} \mathrm{to}$ calculate their $\mathrm{IC}_{50}$ value, which corresponds to the concentration required for $50 \%$ inhibition of cell viability (table 2). Doxorubicin, which is one of the most effective anticancer agents, was used as a reference drug (table 2). Compounds 5c, 7 and 12 revealed potent anti-proliferative effects against A-549 cancer cell line with $\mathrm{IC}_{50}$ of $44.3,17.2$ and $38.7 \mu \mathrm{mol} / \mathrm{l}$, respectively, compare to the reference drug doxorubicin ( $\mathrm{IC}_{50} 48.8 \mu \mathrm{mol} / \mathrm{l}$ ). While compound $5 \mathrm{c}$ was shown to be more potent with $\mathrm{IC}_{50}$ of $5.66 \mu \mathrm{mol} / \mathrm{l}$ against HCT-116 cancer cell line than doxorubicin (IC 50 of $65.1 \mu \mathrm{mol} / \mathrm{l}$ )

Table 1: Anti-proliferative activity of the newly synthesized compounds against human carcinoma cell lines at $100 \mu \mathrm{g} \mathrm{ml}-1$

\begin{tabular}{|c|c|c|c|}
\hline \multirow[t]{2}{*}{ Compounds $^{a}$} & \multicolumn{3}{|c|}{ Inhibition growth (\%) (mean \pm SEM) } \\
\hline & A-549 & HCT-116 & MCF-7 \\
\hline $3 a$ & $59.6 \pm 1.65$ & $28.1 \pm 1.53$ & $10.0 \pm 0.99$ \\
\hline $3 b$ & $2.8 \pm 0.51$ & $39.9 \pm 1.45$ & $0.0 \pm 0.69$ \\
\hline $3 c$ & $72.7 \pm 4.00$ & $47.7 \pm 2.35$ & $11.8 \pm 1.88$ \\
\hline $3 d$ & $70.1 \pm 3.01$ & $48.3 \pm 1.03$ & $60.5 \pm 1.51$ \\
\hline $5 a$ & $12.2 \pm 1.61$ & $38.9 \pm 0.97$ & $3.1 \pm 0.65$ \\
\hline $5 b$ & $20.8 \pm 1.43$ & $41.3 \pm 1.41$ & $33.7 \pm 3.78$ \\
\hline $5 c$ & $95.8 \pm 3.95$ & $83.4 \pm 3.71$ & $81.5 \pm 2.61$ \\
\hline $5 d$ & $44.4 \pm 4.05$ & $37.5 \pm 3.26$ & $14.0 \pm 0.88$ \\
\hline 7 & $92.6 \pm 2.65$ & $92.9 \pm 2.07$ & $73.7 \pm 2.02$ \\
\hline 8 & $13.8 \pm 1.49$ & $42.4 \pm 2.00$ & $3.5 \pm 0.60$ \\
\hline 9 & $75.1 \pm 0.41$ & $44.2 \pm 1.35$ & $6.3 \pm 0.45$ \\
\hline 10 & $62.0 \pm 4.71$ & $48.9 \pm 4.09$ & $8.6 \pm 1.05$ \\
\hline 11 & $15.5 \pm 1.10$ & $33.1 \pm 2.45$ & $0.0 \pm 0.00$ \\
\hline 12 & $92.8 \pm 0.96$ & $70.2 \pm 4.00$ & $29.6 \pm 1.40$ \\
\hline Negative controlb & 0 & 0 & 0 \\
\hline Doxorubicin $^{\mathrm{a}}$ & 100 & 100 & 100 \\
\hline
\end{tabular}

aConcentration of test compounds and positive control (doxorubicin) were $100 \mu \mathrm{g} / \mathrm{ml}$, buntreated cells in DMSO and its final concentration in the cells was less than $0.2 \%, \mathrm{SEM}=$ Standard error mean; each value is the mean of three values

Table 2: IC 50 of the highly antiproliferative active compounds against human cancer cell lines

\begin{tabular}{lll}
\hline Compounds & IC $\mathbf{5}_{\mathbf{0}}(\mathbf{m e a n} \pm \mathbf{S E M})(\boldsymbol{\mu m o l} / \mathbf{l})$ & \\
\cline { 2 - 3 } & $\mathbf{A}-\mathbf{5 4 9}$ & HCT-116 \\
\hline $5 \mathrm{c}$ & $44.3 \pm 057$ & $5.66 \pm 1.50$ \\
7 & $17.2 \pm 2.10$ & - \\
12 & $38.7 \pm 3.01$ & - \\
Doxorubicin & $48.8 \pm 1.28$ & $65.1 \pm 5.15$ \\
\hline
\end{tabular}

$\mathrm{IC}_{50}$ : Compound concentration required to inhibit the cell viability by $50 \%$, SEM = Standard error mean; each value is the mean of three values

\section{Molecular docking study}

In an attempt to rationalize the cytotoxic activity profile exhibited by compounds $5 \mathrm{c}, 7$ and 12 , the molecular docking was studied toward carbonic anhydrase IX (CA IX) (PDB ID: 4BCW) using MOE 2008.10 program. From the data obtained it was found that, in 3D ligand interaction (fig. 1,3 ) compounds 7 and 12 were the most active compounds which exhibited good fitting inside the binding site of the protein molecular surface and having minimum binding energy of-21.03 and-19.69 kJ/mol, respectively in comparison to cocrystallized ligand (TU0) of- $16.35 \mathrm{~kJ} / \mathrm{mol}$ and Rmsd 1.82 (fig. 5). 2D Ligand interaction showed that, compound 7 (fig. 2) formed coordination bond between $\mathrm{Zn}^{++}$and nitrogen atom of pyrazole ring $(2.11 \AA ̊)$ and arene-cation bond between amino acid His64 and 
benzene ring of indole moiety, while compound 12 (fig. 4) formed coordination bond between $\mathrm{Zn}^{++}$and oxygen atom of oxo-pyrimidine (2.12 Å). In comparison to co-crystalline ligand (TU0) which form coordination bond between $\mathrm{Zn}^{++}$and the oxygen atom of $\mathrm{SO}_{2}$ group in addition hydrogen bond between $\mathrm{NH}$ of THr199 and the oxygen atom of $\mathrm{SO}_{2}$ (2.91 Å) (fig. 5, 6). The docking scores of the compounds under study 7 and 12 were observed better than co-crystalline ligand (TU0), which was in agreement with their antiproliferative effects. Our finding is in a similar to that of Güzel et al. 2010 who have been designed a series of 2-(hydrazino carbonyl)-3-

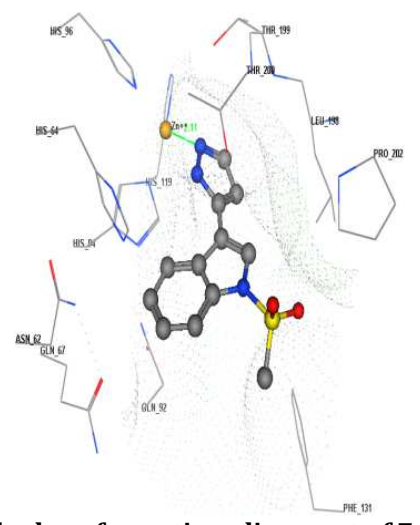

fig. 1: 3D Docked conformation alignment of 7 in the $4 \mathrm{BCW}$ protein binding site

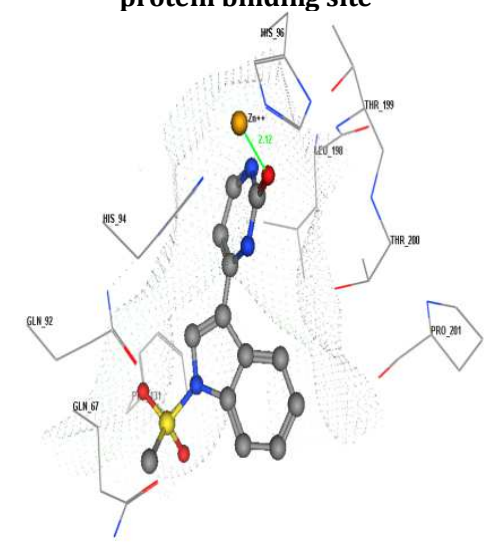

fig. 3: 3D Docked conformation alignment of 12 in the 4BCW protein binding site

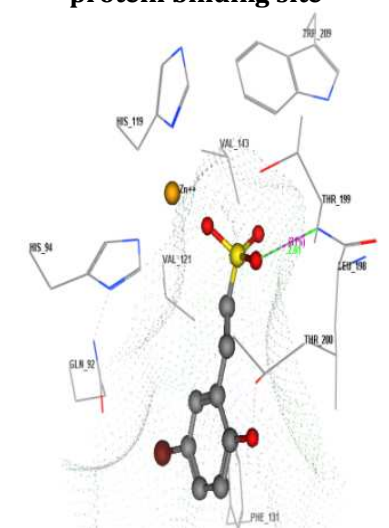

fig. 5: 3D Docked conformation alignment of co-crystalline ligand (TU0) in the 4BCW protein binding site

\section{CONCLUSION}

A new series of pyrazole, pyrimidine, 1,2,3-triazole, thiazolidin-4-one and amino acid derivatives incorporated to 1-methylsulphonyl indole at their 3-positions were prepared. Anti-proliferative activity of some new target compounds was tested in vitro against A-549, HCT-116, substituted-phenyl-1 $\mathrm{H}$-indole-5-sulfonamides as a promising class of carbonic anhydrase inhibitors [38].

Finally, the results of cytotoxic activity and molecular docking suggest that, compounds 5-(1-methylsulphonyl- $1 H$-indol-3-yl)- $1 H$ pyrazole (7) and 6-(1-methylsulphonyl- $1 H$-indol-3-yl)pyrimidin$2(1 H)$-one $(12)$ may have the potential for development as clinical candidates to treat a variety of solid tumors. Also, further work is recommended to confirm the inhibition of carbonic anhydrase IX (CA IX) in a specific bioassay.

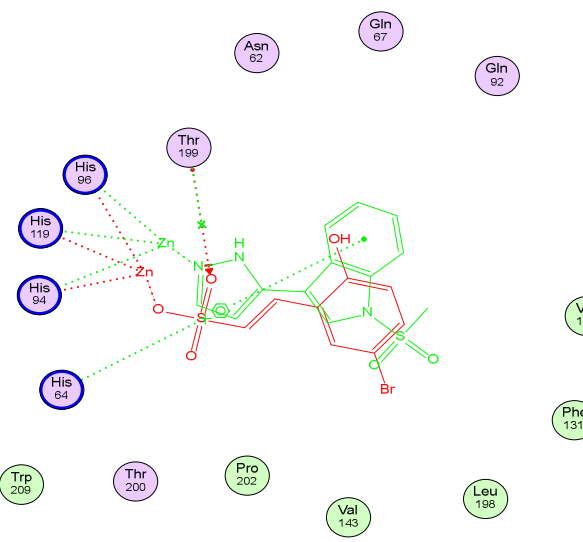

fig. 2: 2D ligand interaction of compound 7 (green) with active site amino acids of $4 \mathrm{BCW}$ compared to the co-crystallized ligand (red)

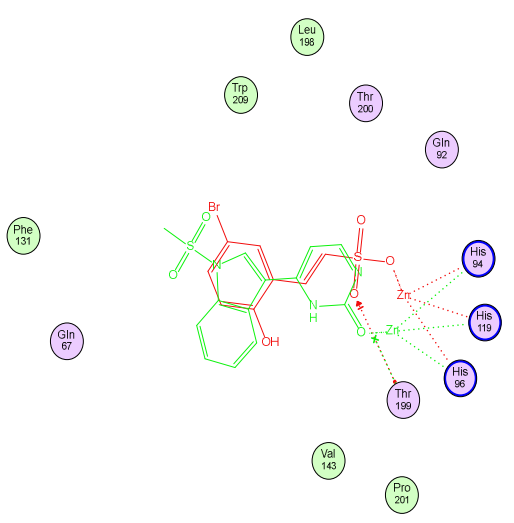

fig. 4: 2D ligand interaction of compound 12 (green) with active site amino acids of $4 \mathrm{BCW}$ compared to the co-crystallized ligand (red)

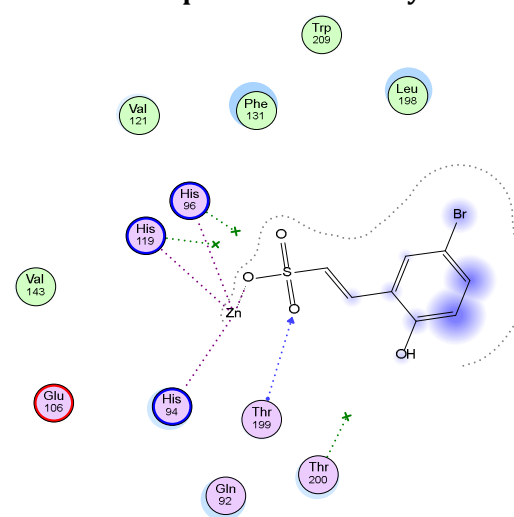

fig. 6: 2D ligand interaction of co-crystalline ligand (TU0) with active site amino acids of $4 \mathrm{BCW}$

and MCF-7cancer cell lines. Pyrazole 5c, 7 and pyrimidine 12 derivatives revealed, potent anti-proliferative effects against A-549 cancer cell line with IC 50 of $44.3,17.2$ and $38.7 \mu \mathrm{mol} / \mathrm{l}$, respectively. Only compound $5 \mathrm{c}$ was shown to be more potent with $\mathrm{IC}_{50}$ of 5.66 $\mu \mathrm{mol} / \mathrm{l}$ against HCT-116 cancer cell line. The model of the interaction of most anti-proliferative compounds 5a, 7 and 12 with the active site 
of carbonic anhydrase IX (PDB ID: 4BCW) was examined via molecular docking. Compounds 7 and 12 were found to be the most active compounds which exhibited good fitting inside the binding site of the protein molecular surface and having minimum binding energy-21.03 and-19.69 $\mathrm{kJ} / \mathrm{mol}$, beside they formed coordination bond between $\mathrm{Zn}^{++}$and nitrogen atom of pyrazole ring $(2.11 \AA \AA)$ or oxygen atom of oxopyrimidine $(2.12 \AA)$, respectively in comparison to co-crystallized ligand (TU0) of- $16.35 \mathrm{~kJ} / \mathrm{mol}$.

\section{ACKNOWLEDGMENT}

The authors are grateful to Kamel H. Shaker and Chemistry Department, Faculty of Science, King Khalid University, Abha, King Saudi Arabia (KSA) for carrying out NMR spectra.

\section{CONFLICTS OF INTERESTS}

\section{Declared none}

\section{REFERENCES}

1. Vilsmeier A, Haack A. About the action of halogen phosphorus to alkyl form anilides: a new method for the preparation of secondary and tertiary $p$-alkylamino-benzaldehyde. Ber 1927;60:119-22.

2. Thomas AD, Asokan CV. Vilsmeier-Haack reactions of carbonyl compounds: synthesis of substituted pyrones and pyridines. Tetrahedron 2004;60:5069-76.

3. Kira MA, Abdel-Rahman M, Gadalla KZ. The vilsmeier haack reaction-III Cyclization of hydrazones to pyrazoles. Tetrahedron Lett 1969;10:109-10.

4. El-Sawy ER, Metwaly AM, Mandour AH. Vilsmeier-Haack Reaction cyclization of N-substituted-3-acetyl indole phenyl hydrazones and their reactions. Egypt J Chem 2007;50:667-81.

5. Quiroga J, Trilleras J, Insuasty B, Abonia R, Nogueras M, Cobo J. Regioselective formulation of pyrazolo $[3,4-b]$ pyridine and pyrazolo[1,5- $a$ ] pyrimidine systems using Vilsmeier-Haack conditions. Tetrahedron Lett 2008;49:2689-91.

6. Damjanovic I, Vukicevic M, Radulovic N, Ellmerer E, Ratkovic Z, Joksovic MD, et al. Synthesis and antimicrobial activity of some new pyrazole derivatives containing a ferrocene unit. Bioorg Med Chem Lett 2009;19:1093-6.

7. Prakash O, Hussain K, Kumar R, Wadhwa D, Sharma C, Aneja KR. A facile iodine(III)-mediated synthesis of 3-(3-aryl-1phenyl-1H-pyrazol-4-yl)-[1,2,4]triazolo[4,3-a]pyridines via oxidation of 2-((3-aryl-1-phenyl-1 $H$-pyrazol-4-yl)methylene)1-(pyridin-2-yl)hydrazines and their antimicrobial evaluations. Org Med Chem Lett 2011;1:5.

8. Meesala R, Nagarajan R. Synthesis of new di heteroaryl carbazoles: a facile and simple route of 3,6-di(pyrazol-4yl)carbazoles. Tetrahedron Lett 2006;47:7557-61.

9. Herbivo C, Comel A, Kirsch G, Raposo MMM. Synthesis of 5-aryl$5^{\prime}$-formyl-2,2'-bithiophenes as new precursors for nonlinear optical (NLO) materials. Tetrahedron 2009;65:2079-86.

10. Paul S, Gupta M, Gupta R, Loupy A. Microwave-assisted solventfree synthesis of pyrazolo[3,4-b]quinolines and pyrazolo[3,4c]pyrazoles using p-TsOH. Tetrahedron Lett 2001;42:3827-9.

11. Park H, Lee K, Park S, Ahn B, Lee J, Cho HY, Lee K. Identification of antitumor activity of pyrazole oxime ethers. Bioorg Med Chem Lett 2005;15:3307-12.

12. Hegab MI, Yousef NM, Nour HF, Ellithey M, Arbid MS. Synthesis and pharmacological activities of some condensed 4-chloro-2,2-dialkyl chromene-3-carbaldehyde derivatives. Acta Pharm 2008;58:15-27.

13. Hegab MI, Abdulla MM. 4-Chloro-2, 2-disubstituted chromen-3carboxaldehyde: synthesis of some fused polycyclic heterocycles as anti-inflammatory, analgesic, anticonvulsant, and antiparkinsonian agents. Arch Pharm Chemi 2006;339:41-7.

14. Naik N, Kumar HV, Shubhavathi T. Synthesis and antioxidant evaluation of novel 5-methoxy indole analogues. Int J Curr Pharm Res 2011;7:109-13.

15. Ramathilagam C, Upgade A, Bhaskar A, Umarani PR, Manivannan V. Synthesis and molecular docking studies of ethyl 1-benzenesulfonyl-2-[(E)-2-(2-methylphenyl) ethenyl] indole-3-carboxylate with human rennin complexed with inhibitor. Asian J Pharm Clin Res 2013;6:96-9.

16. Saravanan B, Upgade A, Bhaskar A, Manivannan V. Synthesis and molecular docking studies of 2 cholromethyl-3-methyl-1- phenyl sulfonyl- $1 h$-indole compound. Asian J Pharm Clin Res 2013;6:262-5.

17. Naim MJ, Alam O, Nawaz F, Alam MJ, Alam P. Current status of pyrazole and it's biological activities. J Pharm Bioallied Sci 2016;8:2-17.

18. Yerragunta V, Suman D, Swamy K, Anusha V, Patil P, Naresh M. Pyrazole and it's biological activity. PharmaTutor Mag 2014;2:40-8.

19. Gupta P, Gupta JK, Bansal S, Halve AK. Synthesis and in vitro antimicrobial studies of some new pyrazolones. Int J Curr Pharm Res 2015;7:25-9.

20. Dansena H, Hj D, Chandrakar K. Pharmacological potentials of pyrimidine derivative: a review. Asian J Pharm Clin Res 2015;8:171-7.

21. Sahu JK, Ganguly S, Kaushik A. Triazoles: a valuable insight into recent developments and biological activities. Chin J Nat Med 2013;11:456-65.

22. Romeo R, Giofrè SV, Carnovale C, Chiacchio MA, Campisi A, Mancuso $\mathrm{R}$, et al. Synthesis and biological activity of triazoleappended N,O-nucleosides. Eur J Org Chem 2014;25:5442-7.

23. Leitans J, Kazaks A, Balode A, Ivanova J, Zalubovskis R, Supuran CT, et al. Efficient expression and crystallization system of cancer-associated carbonic anhydrase isoform IX. J Med Chem 2015;58:9004-9.

24. Lu CL, Lu Z, Li ZC, Gao X, Zhang W. Pharmacophore modeling, virtual screening, and molecular docking studies for the discovery of novel Carbonic anhydrase IX inhibitors. Med Chem Res 2012;21:3417-27.

25. Bashandy MS, Alsaid MS, Arafa RK, Ghorab MM. Design, synthesis and molecular docking of novel $\mathrm{N}, \mathrm{N}$-dimethyl-benzenesulfonamide derivatives as potential antiproliferative agents. J Enzyme Inhib Med Chem 2014;29:619-27.

26. El-Sawy ER, Abo-Salema HM, Yahya SM, Ebaid MS, Mandour AH. Synthesis, cytotoxic, pro-apoptotic evaluation and molecular docking study of some new N-substituted sulphonyl3-indolyl heterocycles. Egypt Pharm J 2015;14:15-29.

27. El-Sawy ER, Abo-Salema HM, Mahmoud K, Zarie ES, ElMetwally AM, Mandour AH. Synthesis, anticancer activity and molecular docking study of novel 1, 3-diheterocycles indole derivatives. Int J Pharm Pharm Sci 2015;7:377-85.

28. El-Sawy ER, Ebaid MS, Abo-Salem HM, El-Hallouty S, Kassem EM, Mandour AH. Synthesis and biological activity of novel series of 4methoxy, and 4,9-dimethoxy-5-substituted furo[2,3-g]-1,2,3benzoxathiazine-7,7-dioxide derivatives. J Adv Res 2014;5:337-46.

29. El-Sawy ER, Mandour AH, Mahmoud K, Islam IE, Abo-Salem HM. Synthesis, antimicrobial and anti-cancer activities of some new $N$-ethyl, $N$-benzyl and $N$-benzoyl-3-indolyl heterocycles. Acta Pharm 2012;62:157-79.

30. El-Sawy ER, Bassyouni FA, Abu-Bakr SH, Rady HM, Abdlla MM. Synthesis and biological activity of some new 1-benzyl and 1benzoyl 3-heterocyclic indole derivatives. Acta Pharm 2010;60:55-71.

31. Mndzhoyan AL, Papayan GL, Zhuruli LD, Karagezyan SG, Galstyan LS, Sarafyan VG. Synthesis and biological study of hydrazine hydrazones of indole aldehydes and ketones series. Arm Khim Zh (USSR) 1969;22:707-13.

32. Mosmann T. Rapid colorimetric assay for cellular growth and survival: application to proliferation and cytotoxicity assays. J Immunol Methods 1983;65:55-63.

33. Tars K, Vullo D, Kazaks A, Leitans J, Lends A, Grandane A, et al. Sulfocoumarins (1,2-benzoxathiine-2,2-dioxides): a class of potent and isoform-selective inhibitors of tumor-associated carbonic anhydrases. J Med Chem 2013;56:293-300.

34. Kaminski G, Jorgensen WL. The performance of the AMBER94, MMFF94 and OPLS-AA force fields for modeling organic liquids. J Phys Chem 1996;100:18010-3.

35. Lensink MF, Méndez R, Wodak SJ. Docking and scoring protein complexes: CAPRI 3rd Edition. Proteins 2007;69:704-18.

36. Mandour AH, El-Sawy ER, Shaker KH, Mustafa MA. Synthesis, antiinflammatory, analgesic and anticonvulsant activities of 1,8dihydro-1-aryl-8-alkyl pyrazolo (3,4-b) indole. Acta Pharm 2010; 60:73-88.

37. El-Sawy ER, Ebaid MS, Abo-Salem HM, Al-Sehemi AG, Mandour AH. Synthesis, anti-inflammatory, analgesic and anticonvulsant 
activities of some new 4,6-dimethoxy-5-(heterocycles) benzofuran starting from naturally occurring visnagin. Arabian J Chem 2014;7:914-23.

38. Güzel O, Innocenti A, Vullo D, Scozzafava A, Supuran CT. 3Phenyl- $1 H$-indole-5-sulfonamides: structure-based drug design of a promising class of carbonic anhydrase inhibitors. Curr Pharm Des 2010;16:3317-26.

\section{How to cite this article}

- Heba M Abo-Salem, Khadiga M Ahmed, Salwa EL-Hallouty, Eslam R EL-Sawy, Adel H Mandour. Synthesis, molecular docking and anti-proliferative activity of new series of 1methylsulphonyl-3-indolyl heterocycles. Int J Pharm Pharm Sci 2016;8(12):113-123. 